DAMTP/98-118

\title{
TWISTING OF QUANTUM DIFFERENTIALS AND THE PLANCK SCALE HOPF ALGEBRA
}

\author{
Shahn Majid円+ Robert Oeckl \\ Department of Applied Mathematics \& Theoretical Physics \\ University of Cambridge, Cambridge CB3 9EW
}

October, 1998

\begin{abstract}
We show that the crossed modules and bicovariant different calculi on two Hopf algebras related by a cocycle twist are in 1-1 correspondence. In particular, for quantum groups which are cocycle deformation-quantisations of classical groups the calculi are obtained as deformation-quantisation of the classical ones. As an application, we classify all bicovariant differential calculi on the Planck scale Hopf algebra $\mathbb{C}[x] \aleph_{\hbar, G} \mathbb{C}[p]$. This is a quantum group which has an $\hbar \rightarrow 0$ limit as the functions on a classical but non-Abelian group and a $G \rightarrow 0$ limit as flat space quantum mechanics. We further study the noncommutative differential geometry and Fourier theory for this Hopf algebra as a toy model for Planck scale physics. The Fourier theory implements a T-duality like self-duality. The noncommutative geometry turns out to be singular when $G \rightarrow 0$ and is therefore not visible in flat space quantum mechanics alone.
\end{abstract}

Keywords: exterior algebra, bicrossproduct, Radford-Drinfeld-Yetter module, quantum double, quantum gravity, Moyal product, Born reciprocity, T-duality, deformation quantisation.

\section{Introduction}

Recent years have seen considerable advances in the noncommutative geometry related to quantum groups, including a more or less complete theory of quantum bundles and connections with quantum group structure, quantum homogeneous spaces, etc. Particularly important for all these constructions is the differential calculus or bimodule $\Omega^{1}$ of 1 -forms on the 'quantum space'. Recently, the translation-bicovariant calculi on the quantum group itself have been classified for the class of 'factorisable' quantum groups [1]. Using different methods, one also has a classification of the calculi on bicrossproduct Hopf algebras $\mathbb{C}(M) \bowtie \mathbb{C} G$ associated to finite group factorisations [2].

In this paper we present a third and novel 'deformation theoretic' approach to the construction of differential calculi which works for quantum groups which are cocycle deformationquantisations [3] of classical groups. We show that the calculi on the quantum group are in correspondence with the calculi on the classical group and we provide the explicit deformationquantisation of the latter to obtain the former. This includes the large class of quantum groups related to triangular solutions of the Yang-Baxter equation [3], not covered by the previous approaches.

\footnotetext{
${ }^{1}$ Royal Society University Research Fellow and Fellow of Pembroke College, Cambridge
} 
The paper consists of a general functorial result (in Section 3) concerning how the construction of quantum differential calculi on a quantum group responds under a certain 'cotwisting' or 'gauge equivalence' 四 5 operation in the category of quantum groups. The main idea of this cotwisting is to start with an initial Hopf algebra $H$, e.g. a commutative one (the coordinate ring of a classical group) and modify its product by a cocycle $\chi$ to a new quantum group $H^{\chi}$. We show that the exterior algebra of the quantum differential calculus likewise twists as a super-Hopf algebra. This is the main result of Section 2.

The main technical result is the construction of a nontrivial monoidal functor relating the bicovariant bimodules over the twisted and untwisted Hopf algebras. Moreover, bicovariant bimodules over a Hopf algebra $H$ are equivalent to the representations of the quantum double $D(H)$ or, equivalently, to the crossed modules (Drinfeld-Radford-Yetter modules) ${ }_{H}^{H} \dot{\mathcal{M}}$ associated to $H$. Therefore a corollary, also in Section 2, is the construction of a nontrivial monoidal functor

$$
\mathcal{F}^{\chi}:{ }_{H}^{H} \dot{\mathcal{M}} \rightarrow{ }_{H}^{H^{\chi}} \dot{\mathcal{M}}
$$

or equivalently (by Tannaka-Krein reconstruction) of an isomorphism

$$
D\left(H^{\chi}\right) \cong D(H)_{\tilde{\chi}}
$$

(for twisting of the coproduct by a certain other cocycle $\tilde{\chi}$ in $D(H)$ ).

Finally, in Section 3, we add the consideration of the exterior differential to complete the general theory. As a further nontrivial corollary related to the particular crossed modules needed for quantum differential calculi, we obtain an identification (via the above functor) of the quantum adjoint action under the twisted and untwisted Hopf algebras, extending a result in [6].

The second half of the paper consists of the application of these general results to a particular Hopf algebra $\mathbb{C}[x] \bowtie_{\hbar, \mathrm{G}} \mathbb{C}[p]$ which was introduced 10 years ago as a new 'Hopf algebra approach' to Planck scale physics [7]. It appears to be the first serious attempt to develop gravitationally-modified quantum mechanics as 'noncommutative geometry' by quantum group methods, namely by modifying the usual $[x, p]=\imath \hbar$ commutation relations in such a way as to allow a coproduct. Thus, the Planck scale Hopf algebra has two parameters $\hbar, \mathrm{G}$ and the Hopf algebra structure

$$
[x, p]=\imath \hbar\left(1-e^{-\frac{x}{\mathrm{G}}}\right), \quad \Delta x=x \otimes 1+1 \otimes x, \quad \Delta p=p \otimes e^{-\frac{x}{\mathrm{G}}}+1 \otimes p .
$$

In the limit $\hbar \rightarrow 0$ one obtains the classical ring $C\left(B_{+}\right)$of functions on a group $B_{+}=\mathbb{R} \ltimes{ }_{\mathrm{G}} \mathbb{R}$ (a non-Abelian but classical group as the classical phase space) and in another limit $G \rightarrow 0$ one obtains the usual flat space Heisenberg algebra when restricted to $x>0$ (the coalgebra is singular in this limit). Here $\hbar$ and $G$ are arbitrary constants, but the latter plays a role similar to (a dimensionful multiple of) the gravitational Newton coupling constant when one compares free particle motion with the motion of a particle falling into a black hole [7] [5]. More precisely, $\mathrm{G}$ plays the role of $\mathrm{G}_{\text {Newton }} M / c^{2}$ where $M$ is the mass of the black hole and $c$ is the speed of light. Thus we can also consider $\mathrm{G}$ more physically as playing the role of the gravitational mass or radius of curvature of the background geometry.

A main feature of this Planck scale Hopf algebra is its self-duality, i.e. the linear functionals on this quantum system (containing the states) can be convolved and as such form an isomorphic Hopf algebra which can be viewed as the observables of a dual quantum system (the original algebra of observables containing the states of this). It seems likely that more recent constructions of $T$-duality in string theory can be viewed as generalisations to string theory of such a duality. On the other hand, the noncommutative differential geometry of the Planck scale Hopf 
algebra could not be explored 10 years ago; we are able to do this now. While we recover the known differential calculi on $B_{+}$as $\hbar \rightarrow 0$, the differential calculi are equally valid for general $\hbar$ and thereby extend our geometrical notions to the quantum system. As well as the the classical and flat space quantum mechanical limits there is a third limit where $\imath \kappa=\mathrm{G} / \hbar$ is held fixed as $\hbar, \mathrm{G} \rightarrow \infty$, and the Planck scale Hopf algebra tends to the enveloping algebra $U\left(\mathfrak{b}_{-}\right)\left(\mathfrak{b}_{-}\right.$the opposite of the Lie algebra $\mathfrak{b}_{+}$of $\left.B_{+}\right)$. Such an algebra has been proposed as a noncommutative model of spacetime (here in 2 dimensions) covariant under the $\kappa$-Poincaré quantum group [8]; its noncommutative geometry can therefore be obtained as a special case.

It is known that the Planck scale Hopf algebra is a cocycle twist of $U\left(\mathfrak{b}_{+}\right)$. In view of its self-duality this implies that it is also a cotwist deformation quantisation of $C\left(B_{+}\right)$, where $B_{+}$ is the group above. We begin this part of the paper in Section 4 by obtaining the cotwisting cocycle $\chi_{\hbar}$ explicitly. In this way we have explicitly the deformation-quantisation which undoes the $\hbar \rightarrow 0$ limit above as a switching on of the cocycle $\chi_{\hbar}$. The cocycle $\chi_{\hbar}$ has in fact a similar form to the operation in the Moyal product [9], i.e. this is a variant of the Moyal product or *-product quantisation, the variation being that we do not start from $\mathbb{R}^{2}$ but from a non-Abelian (i.e. in some sense 'curved') classical group manifold $B_{+}$. On the other hand, the first order differential calculi on $C\left(B_{+}\right)$have been completely classified recently in [10 and hence our general construction in Section 3 provides, in Section 4, a functorial 'deformation-quantisation' of these to calculi on the Planck scale Hopf algebra.

We then compute the entire quantum exterior algebra in the case of the quantisation of the standard classical 2-dimensional differential calculus on $B_{+}$. We also provide some elements of quantum Poisson theory in this case, including quantum-geometric Hamilton equations of motion. Remarkably, the exterior algebra and this quantum-geometrical picture is highly singular when $G \rightarrow 0$, i.e. is not visible in flat space quantum mechanics alone. As in [7], we conclude that the presence of even a small amount of 'gravity' makes quantum mechanics better behaved and restores its geometry.

The remaining Section 5 completes the noncommutative picture with formulae for the left and right invariant integrals, exponentials and the Fourier transform on the Planck scale Hopf algebra. In fact the quantum Fourier transform is a linear isomorphism

$$
\mathcal{T}: \mathbb{C}[x] \bowtie_{\hbar, \mathrm{G}} \mathbb{C}[p] \rightarrow \mathbb{C}[\bar{p}] \triangleright_{\frac{1}{\hbar}, \frac{\mathrm{G}}{\hbar}} \mathbb{C}[\bar{x}],
$$

which interchanges the roles of $x, p$ (a version of Born reciprocity) and at the same time requires inversion of $\hbar$. Thus we see that it explicitly implements the T-duality-like feature of our toy model of Planck scale physics in [7]. This self-duality isomorphism is singular when $\hbar \rightarrow 0$, i.e. only visible due to the presence of quantisation. When $\hbar \rightarrow 0$, one obtains instead a non-Abelian Fourier isomorphism between two completely different objects, namely

$$
\mathcal{T}: C\left(B_{+}\right) \rightarrow U\left(\mathfrak{b}_{+}\right) .
$$

The right hand side here is also a version of $\kappa$-Minkowski space, with $\imath \kappa=\mathrm{G}$, so Fourier theory on this is recovered in the classical limit.

\section{Preliminaries}

The general results in Sections 2,3 work over a general field $k$. The application results in Sections 4,5 are over $k=\mathbb{C}$. We use the usual notations for a Hopf algebra $(H, \Delta, S, \epsilon)$ where $H$ is a unital algebra, $\Delta: H \rightarrow H \otimes H$ and $\epsilon: H \rightarrow k$ the counital coalgebra and $S: H \rightarrow H$ the antipode or generalised 'inversion'. For convenience we require that $S$ is invertible. We use the 
Sweedler notation $\Delta h=h_{(1)} \otimes h_{(2)}$ for $h \in H$ and similarly for a coaction $\beta: V \rightarrow H \otimes V$ we use the notation $\beta(v)=v_{(1)} \otimes v_{(\infty)}$. Here coproducts and coactions obey axioms like products and actions, but with arrows reversed, see [5] for an introduction.

Given a Hopf algebra $H$ and invertible $\chi \in H \otimes H$ a cocycle in the sense

$$
\chi_{23}(\mathrm{id} \otimes \Delta \chi)=\chi_{12}(\Delta \otimes \mathrm{id}) \chi, \quad(\epsilon \otimes \mathrm{id}) \chi=1,
$$

one has a new Hopf algebra $H_{\chi}$ with the same algebra structure and counit as $H$ and the new coproduct and antipode

$$
\Delta_{\chi}=\chi \Delta() \chi^{-1}, \quad S_{\chi}=U S() U^{-1}, \quad U=\chi^{(1)}\left(S \chi^{(2)}\right) .
$$

This is the twist of $H$, see [4] [6]. Dually, given a Hopf algebra $H$ and $\chi: H \otimes H \rightarrow k$ convolution invertible, a cocycle in the sense

$$
\chi\left(g_{(1)} \otimes f_{(1)}\right) \chi\left(h \otimes g_{(2)} f_{(2)}\right)=\chi\left(h_{(1)} \otimes g_{(1)}\right) \chi\left(h_{(2)} g_{(2)} \otimes f\right), \quad \chi(1 \otimes h)=\epsilon(h)
$$

one has a new Hopf algebra $H^{\chi}$ with the same coalgebra and unit as $H$ and the new product and antipode

$h \bullet g=\chi\left(h_{(1)} \otimes g_{(1)}\right) h_{(2)} g_{(2)} \chi^{-1}\left(h_{(3)} \otimes g_{(3)}\right), \quad S^{\chi} h=U\left(h_{(1)}\right) S h_{(2)} U^{-1}\left(h_{(3)}\right), \quad U(h)=\chi\left(h_{(1)} \otimes S h_{(2)}\right)$.

See [5].

Next, given a Hopf algebra (with invertible antipode), one has a braided category ${ }_{H}^{H} \dot{\mathcal{M}}$ of crossed modules 11] 12] 113. Objects are vector spaces $V$ which are both $H$-modules and $H$ comodules. The two structures obey a compatibility condition. This and the braiding $\Psi_{V, W}$ : $V \otimes W \rightarrow W \otimes V$ are

$$
h_{(1)} v_{(1)} \otimes h_{(2)} \triangleright v_{(\infty)}=\left(h_{(1)} \triangleright v\right)_{(1)} h_{(2)} \otimes\left(h_{(1)} \triangleright v\right)_{(\infty)}, \quad \Psi_{V, W}(v \otimes w)=v_{(1)} \triangleright w \otimes v_{(\infty)},
$$

where $\triangleright$ denotes the action. This is a slight reformulation (in a completely standard manner) of the braided category of modules over the Drinfeld quantum double $D(H)$ (here $D(H)=H^{* o \mathrm{op}} \bowtie H$ generated when $H$ is finite-dimensional by $H$ and $H^{* o p}$ as sub-Hopf algebras and an $H^{* o p_{-}}$ module structure is equivalent to a $H$-comodule structure; the latter formulation then avoids finite-dimensionality). The cross relations in $D(H)$ are

$$
h \phi=\phi_{(2)} h_{(2)}\left\langle S h_{(1)}, \phi_{(1)}\right\rangle\left\langle h_{(3)}, \phi_{(3)}\right\rangle, \quad \forall h \in H, \quad \phi \in H^{*} .
$$

$H$ is a crossed module over itself with action by left-multiplication and the adjoint coaction

$$
\beta(h)=h_{(1)} S h_{(3)} \otimes h_{(2)} .
$$

Here, $\operatorname{ker} \epsilon$ is a sub-crossed module.

A differential calculus over any algebra $H$ means a specification of an $H$-bimodule of differential 1-forms $\Omega^{1}$ and a map d $: H \rightarrow \Omega^{1}$ obeying the Leibniz rule

$$
\mathrm{d}(h g)=(\mathrm{d} h) g+h \mathrm{~d} g
$$

and surjective in the sense $\Omega^{1}=\operatorname{span}\{h \mathrm{~d} g \mid h, g \in H\}$. Such 1-forms can then be extended to an entire exterior algebra with $\mathrm{d}^{2}=0$, although not necessarily uniquely. When $H$ is a Hopf algebra it is natural to require that $\Omega^{1}$ is covariant under left and right coactions of $H$ [14]. 
Thus we require $\Omega^{1}$ to be both a bimodule and a bicomodule, with the coactions bimodule maps and mutually commuting, i.e. $\Omega^{1} \in{ }_{H}^{H} \mathcal{M}_{H}^{H}$, the category of bicovariant bimodules over $H$. It is known that bicovariant bimodules are equivalent to crossed modules under the correspondence $\Omega^{1}=V \otimes H$ for some $V \in{ }_{H}^{H} \dot{\mathcal{M}}$. The bimodule structure on $\Omega^{1}$ is

$$
h \cdot(v \otimes g)=h_{(1)} \triangleright v \otimes h_{(2)} g, \quad(v \otimes g) \cdot h=v \otimes g h .
$$

The left and right coactions are likewise induced from $V$ and $H$ using the given coaction on $V$ and the coproduct of $H$. The remaining axioms of a differential calculus then reduce to $V$ a quotient of ker $\epsilon \in{ }_{H}^{H} \dot{\mathcal{M}}$. The corresponding $\mathrm{d}$ is

$$
\mathrm{d} h=(\pi \otimes \mathrm{id})\left(h_{(1)} \otimes h_{(2)}-1 \otimes h\right),
$$

where $\pi$ is the projection from ker $\epsilon$ to $V$. The space $V$ is the space of right-invariant differential forms.

Moreover, given $\Omega^{1}$ there is its maximal prolongation differential graded algebra $\widetilde{\Omega}=\oplus_{n} \Omega^{n}$ where $\Omega^{0}=H, \Omega^{1}=\Omega^{1}$ and $\Omega^{n}$ is a certain quotient of $\Omega^{1} \otimes_{H} \cdots \otimes_{H} \Omega^{1}$ (n-fold). The product is given by $\otimes_{H}$ or with degree 0 by the bimodule structure. The differential structure is $\mathrm{d}$ extended by $\mathrm{d}^{2}=0$ and the graded Leibniz rule. The quotienting in $\widetilde{\Omega}$ is the minimum required for the extension of $\mathrm{d}$. There is also a Woronowicz prolongation differential graded algebra $\widehat{\Omega}$. which is likewise a quotient of the tensor algebra, this time using the braiding induced from ${ }_{H}^{H} \dot{\mathcal{M}}$ to 'skew-symmetrise'. It is a quotient of the maximal prolongation, and for q-deformed examples is typically a q-deformation of the classical exterior algebra.

Finally, dual to the vector space $V$ corresponding to a bicovariant calculus on $H$ is a subspace $L \subseteq \operatorname{ker} \epsilon \subset U$ of a Hopf algebra $U$ dual to $H$. We have a self-contained notion of 'space of rightinvariant vector fields' $L \subseteq \operatorname{ker} \epsilon \in \in_{U}^{U} \dot{\mathcal{M}}$, where $\operatorname{ker} \epsilon \subset U$ is a crossed module by the coproduct and the quantum adjoint action. The latter restricts to a map $L \otimes L \rightarrow L$ with the result that $L$ is sometimes called a 'quantum Lie algebra'.

Moreover, any $x \in L$ acts on $H$ by $\tilde{x}(h)=\left\langle x, h_{(1)}\right\rangle h_{(2)}$, say. One may view both $H$ (as above) and $L=V^{*}$ in the braided category ${ }_{H}^{H} \dot{\mathcal{M}}$, where $L$ is in this category by

$$
h \triangleright x=\left\langle S h, x_{(1)}\right\rangle x_{(2)}, \quad\left\langle\phi, x_{(1)}\right\rangle x_{(\infty)}=\left(S^{-1} \phi_{(2)}\right) x \phi_{(1)}
$$

for $h \in H$ and $\phi \in U$ (the coregular action and coadjoint coaction). Then one has the braidedLeibniz rule

$$
\tilde{x}(h g)=(\tilde{x}(h)) g+h_{i} \tilde{x^{i}}(g)
$$

where $\Psi^{-1}(x \otimes h) \equiv h_{i} \otimes x^{i}$ (sum over the index) is the inverse of the braiding between $L, H$, cf. [1] in different conventions. Thus the elements of $L$ indeed act as braided derivations or 'vector fields'. To work with them we will employ the following convenient notation: if $\{v, w, \cdots\}$ is a given basis of $V$ with dual basis $\left\{v^{*}, w^{*}, \cdots\right\}$ say, we denote the braided vector field $\widetilde{v^{*}}$ by $\partial_{v}$, etc. One has $\mathrm{d} h=v \partial_{v}(h)+w \partial_{w}(h)+\cdots$ (sum over the basis).

\section{Twisting of bicovariant bimodules and crossed modules}

In this section we study the response of the category of bicovariant bimodules and the category of crossed modules of a Hopf algebra $H$ under twisting. We will later apply this (in Section 3 ) to obtain the response under twisting of a quantum differential calculus $\Omega^{1}$ and its exterior algebra. 


\subsection{Bicovariant bimodules and tensor algebras}

Let $H$ be a Hopf algebra. We recall from the Preliminaries that a bicovariant bimodule $\Omega$ means an $H$-bimodule and $H$-bicomodule where the coactions are each bimodule maps. We denote the coactions by

$$
\beta_{L}(\alpha)=\alpha_{(1)} \otimes \alpha_{(\infty)}, \quad \beta_{R}(\alpha)=\alpha_{(0)} \otimes \alpha_{(1)} .
$$

Since these coactions commute, we write

$$
\alpha_{(1)} \otimes \alpha_{(\infty)(0)} \otimes \alpha_{(\infty)(1)}=\alpha_{(0)(1)} \otimes \alpha_{(0)(\infty)} \otimes \alpha_{(0)}=\alpha_{(1)} \otimes \alpha_{(\underline{2})} \otimes \alpha_{(3)}
$$

when both are applied. Here the underline marking the component living in $\Omega$ is an alternative (but unconventional) notation for coactions which allows renumberings in the same manner as for coproducts.

It is known (Brzezinski's theorem) that the Woronowicz exterior algebra is a super-Hopf algebra [15]. This is also known (the case given in detail in [15]) for a similar exterior algebra where the quotient is generated in degree 2. We begin with an analogous result for the tensor algebra on $\Omega$ in a slightly more general form as a braided group. The proof is analogous to these known results and hence we give it here only in outline form. We also recall that the category of $\mathbb{Z}$-graded spaces is braided with braiding

$$
\Psi(\alpha \otimes \beta)=q^{|\alpha||\beta|} \beta \otimes \alpha
$$

for any invertible $q$. It can be identified as the the category of comodules under a quantum group $\mathbb{Z}_{q}$ [5]. We denote by $\Omega^{\otimes_{H}}{ }^{n}$ the n-fold tensor product $\Omega \otimes_{H} \cdots \otimes_{H} \Omega$.

Proposition $2.1 \mathrm{cf}$. [15] Let $\Omega$ be an $H$-bicovariant bimodule. The tensor algebra $T_{q} \Omega=$ $\oplus_{n} \Omega^{\otimes_{H} n}$ is a braided group in the category of $\mathbb{Z}_{q}$-comodules. The coproduct and antipode are

$$
\Delta=\beta_{L}+\beta_{R}, \quad S \alpha=-\left(S \alpha_{(1)}\right) \cdot \alpha_{(\underline{2})} \cdot\left(S \alpha_{(3)}\right)
$$

on degree 1 and extended to $T_{q} \Omega$ as a braided group.

Proof The proof is by induction. First note that $\Delta$ as stated is a bimodule map since $\beta_{L}, \beta_{R}$ are. We extend it by

$$
\Delta(\alpha \underset{H}{\otimes} \beta)=q^{\left|\alpha_{(2)}\right|\left|\beta_{(1)}\right|} \alpha_{(1)} \underset{H}{\otimes} \beta_{(1)} \otimes \alpha_{(2)} \underset{H}{\otimes} \beta_{(2)}
$$

which is well-defined since $\Delta$ on $\alpha, \beta$ is a bimodule map. Moreover, for the same reason $\Delta$ remains a bimodule map. Coassociativity on degree 1 follows from that of $H$ and the bicomodule properties of $\Omega$ (this step is the same as in 15]), and likewise extends to all degrees by induction. By construction, $\Delta$ is an algebra map with the braided tensor product in the category of $\mathbb{Z}_{q^{-}}$ comodules. Hence we have a bialgebra in the category of $\mathbb{Z}_{q}$-comodules.

Similarly, it is easy to see from $\beta_{L}, \beta_{R}$ bimodule maps that $S(h \cdot \alpha)=(S \alpha) \cdot S h$ and $S(\alpha \cdot$ $h)=(S h) \cdot S \alpha\left(S\right.$ a skew-bimodule map). We extend $S$ to higher products by $S\left(\alpha \otimes_{H} \beta\right)=$ $q^{|\alpha||\beta|}(S \beta) \otimes_{H}(S \alpha)$ which is therefore well defined and remains a skew-bimodule map. That the antipode axiom is fulfilled then only has to be verified on degree 1 , and extends by induction to all degrees. This is easily verified and is the same as in [15].

The structure of $T_{q} \Omega$ as a bicovariant bimodule can be recovered from the bialgebra structure in the following way: The module structure is given by the multiplication with one factor in $H$. The comodule structure is given by the coproduct with subsequent projection of the respective component to $H$ (the degree zero part). 
Lemma 2.2 Let $\chi \in H \otimes H$ be a counital 2-cocycle for $H$. Then $\chi$ extends to a graded counital 2-cocycle $\chi \in T_{q} \Omega \otimes T_{q} \Omega$ by inclusion. The twisting of the coproduct yields $\left(T_{q} \Omega\right)_{\chi}$ as a bicovariant bimodule over $H_{\chi}$ and a $\mathbb{Z}_{q}$-braided group (a Hopf algebra in the category of $\mathbb{Z}_{q}$-comodules). In particular, the degree 0 part of $\left(T_{q} \Omega\right)_{\chi}$ is $H_{\chi}$.

Proof The cocycle is embedded in degree 0 and the braiding with degree 0 in the category of $\mathbb{Z}_{q}$-comodules is the trivial one (independent of $q$ ). Hence for this special type of cocycle the requirements to make $T_{q} \Omega$ a $\mathbb{Z}_{q}$-braided group have the same form as for bosonic Hopf algebras. This cocycle condition then reduces on $\chi$ to the usual cocycle condition for the construction of $H_{\chi}$. Also, the twisting preserves degree, and is thus a functor in the category of $\mathbb{Z}_{q}$-comodules. As for $T_{q} \Omega$, we recover the bicovariant bimodule structure of the twisted objects by using the product and the coproduct with subsequent projection.

The restriction of this Lemma to $\Omega \subset T_{q} \Omega$ provides the desired twist of bicovariant bimodules. That this gives rise to an isomorphism of categories is the following theorem.

Theorem 2.3 Let $H$ be a Hopf algebra, $\chi \in H \otimes H$ a counital 2-cocycle. There is an isomorphism of braided categories $\mathcal{G}_{\chi}:{ }_{H}^{H} \mathcal{M}_{H}^{H} \rightarrow{ }_{H_{\chi}}^{H_{\chi}} \mathcal{M}_{H_{\chi}}^{H_{\chi}}$. $\mathcal{G}_{\chi}$ leaves the actions unchanged and transforms the coactions according to

$$
\left(\beta_{L}\right)_{\chi}=\chi \beta_{L} \chi^{-1}, \quad\left(\beta_{R}\right)_{\chi}=\chi \beta_{R} \chi^{-1}
$$

The monoidal structure is provided by the (identity) natural transformation

$$
c_{\chi}: \mathcal{G}_{\chi}(V) \otimes_{H_{\chi}} \mathcal{G}_{\chi}(W) \rightarrow \mathcal{G}_{\chi}\left(V \otimes_{H} W\right), \quad v \otimes_{H_{\chi}} w \mapsto v \otimes_{H} w
$$

for all $V, W \in{ }_{H}^{H} \mathcal{M}_{H}^{H}$.

Proof In view of the proposition above, we write actions as multiplications $a v \equiv a \cdot v$, $a \bullet v \equiv a \cdot \chi_{v}$ (and similarly for the right actions). By restricting Lemma 2.2 to degree 1 we already know that $\mathcal{G}_{\chi}$ maps bicovariant bimodules to bicovariant bimodules. To see that $\mathcal{G}_{\chi}$ is indeed a functor we have to show that it maps morphisms to morphisms. Let $f: V \rightarrow W$ be a morphism in ${ }_{H}^{H} \mathcal{M}_{H}^{H}$. Since $\mathcal{G}_{\chi}$ leaves the actions invariant, we just have to check that $f$ remains a comodule map. For the left coaction,

$$
\left(\beta_{L}\right)_{\chi} \circ f=\chi\left(\beta_{L} \circ f\right) \chi^{-1}=\chi\left((\mathrm{id} \otimes f) \circ \beta_{L}\right) \chi^{-1}=(\mathrm{id} \otimes f) \circ\left(\chi \beta_{L} \chi^{-1}\right)=(\mathrm{id} \otimes f) \circ\left(\beta_{L}\right)_{\chi} .
$$

Accordingly for the right coaction.

Next, we show that $\mathcal{G}_{\chi}$ is monoidal. The only non-trivial part is to check that $c_{\chi}$ is a morphism in ${ }_{H_{\chi}}^{H_{\chi}} \mathcal{M}_{H_{\chi}}^{H_{\chi}}$. We only do the proof for the left action and left coaction; the right handed cases follow by symmetry. For the left action this is the commutativity of the diagram

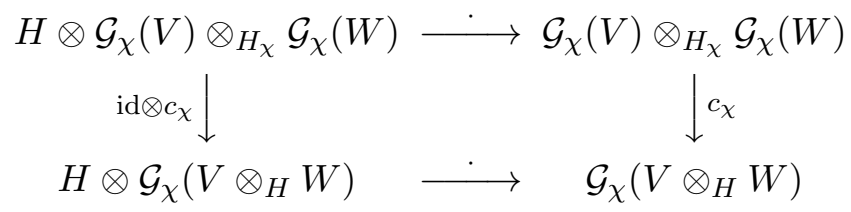

which is evident since

$$
c_{\chi}\left(h\left(v \otimes_{H_{\chi}} w\right)\right)=c_{\chi}\left(h v \otimes_{H_{\chi}} w\right)=h v \otimes_{H} w=h\left(v \otimes_{H} w\right)=h c_{\chi}\left(v \otimes_{H_{\chi}} w\right) .
$$


For the left coaction the diagram is

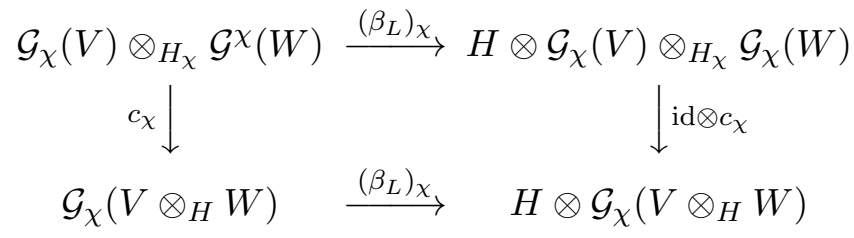

We write the twisted coaction as $\left(\beta_{L}\right)_{\chi}(v)=v_{<1>} \otimes v_{<\underline{2}>}$ for clarity, and we denote a second copy of $\chi$ by $\chi^{\prime}$. Then,

$$
\begin{aligned}
\left(\beta_{L}\right)_{\chi} \circ c_{\chi}\left(v \otimes_{H_{\chi}} w\right) & =\left(\beta_{L}\right)_{\chi}\left(v \otimes_{H} w\right)=\chi \beta_{L}\left(v \otimes_{H} w\right) \chi^{-1} \\
& =\chi^{(1)} v_{(1)} w_{(1)} \chi^{-(1)} \otimes \chi^{(2)}\left(v_{(\underline{2})} \otimes_{H} w_{(\underline{2})}\right) \chi^{-(2)} \\
& =\chi^{(1)} v_{(1)} w_{(1)} \chi^{-(1)} \otimes \chi^{(2)} v_{(\underline{2})} \otimes_{H} w_{(\underline{2})} \chi^{-(2)} \\
& =\chi^{(1)} v_{(1)} \chi^{\prime(1)} \chi^{(1)} w_{(1)} \chi^{-(1)} \otimes \chi^{(2)} v_{(\underline{2})} \chi^{\prime-(2)} \chi^{(2)} \otimes_{H} w_{(\underline{2})} \chi^{-(2)} \\
& =\chi^{(1)} v_{(1)} \chi^{\prime-(1)} \chi^{\prime(1)} w_{(1)} \chi^{-(1)} \otimes \chi^{(2)} v_{(\underline{2})} \chi^{\prime-(2)} \otimes_{H} \chi^{\prime(2)} w_{(\underline{2})} \chi^{-(2)} \\
& =v_{<1>} w_{<1>} \otimes v_{<\underline{2}>} \otimes_{H} w_{<\underline{2}>} \\
& =\left(\mathrm{id} \otimes c_{\chi}\right)\left(v_{<1>} w_{<1>} \otimes v_{<\underline{2}>} \otimes_{H_{\chi}} w_{<\underline{2}>}\right) \\
& =\left(\mathrm{id} \otimes c_{\chi}\right) \circ\left(\beta_{L}\right)_{\chi}\left(v \otimes_{H_{\chi}} w\right) .
\end{aligned}
$$

For the braiding we have to show that the following digram commutes

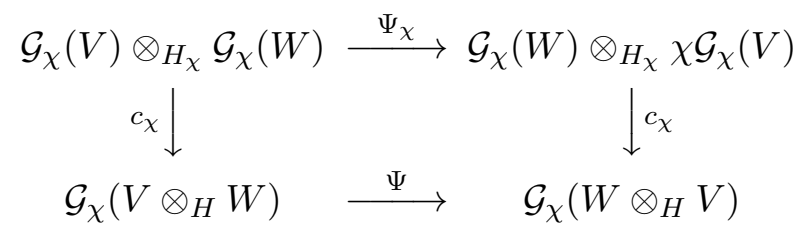

Since $c_{\chi}$ is the identity transformation, this means that the braiding in the untwisted and twisted category should be the same. This is,

$$
\begin{aligned}
& c_{\chi} \circ \Psi_{\chi}\left(v \otimes_{H_{\chi}} w\right) \\
& =v_{<1>} w_{<\underline{1}>} S_{\chi} w_{<2>} \otimes_{H} S_{\chi} v_{<2>} v_{<\underline{3}>} w_{<3>} \\
& =v_{<1>} w_{<\underline{1}>} S_{\chi}\left(v_{<2>} w_{<2>}\right) \otimes_{H} v_{<\underline{3}>} w_{<3>} \\
& =\chi^{(1)} v_{(1)} w_{(\underline{1})} \chi^{-(1)} S_{\chi}\left(\chi^{(2)} v_{(2)} w_{(2)} \chi^{-(2)}\right) \otimes_{H} \chi^{(3)} v_{(\underline{3})} w_{(3)} \chi^{-(3)} \\
& =\chi^{(1)} v_{(1)} w_{(\underline{1})} \chi^{-(1)} \chi^{\prime(1)} S \chi^{(2)} S\left(\chi^{(2)} v_{(2)} w_{(2)} \chi^{-(2)}\right) S \chi^{\prime \prime-(1)} \chi^{\prime \prime-(2)} \otimes_{H} \chi^{(3)} v_{(\underline{3})} w_{(3)} \chi^{-(3)} \\
& =\chi^{(1)} v_{(1)} w_{(\underline{1})} \chi^{-(1)} \chi^{\prime(1)} S\left(\chi^{-(2)} \chi^{\prime(2)}\right) S\left(v_{(2)} w_{(2)}\right) \otimes_{H} S\left(\chi^{\prime \prime-(1)} \chi^{(2)}\right) \chi^{\prime \prime-(2)} \chi^{(3)} v_{(\underline{3})} w_{(3)} \chi^{-(3)} \\
& =\chi^{(1)} v_{(1)} w_{(\underline{1})} \chi^{-(1)}{ }_{(1)} S\left(\chi_{(2)}^{-(1)}\right) S\left(v_{(2)} w_{(2)}\right) \otimes_{H} S\left(\chi_{(1)}^{(2)}\right) \chi_{(2)}^{(2)} v_{(\underline{3})} w_{(3)} \chi^{-(2)} \\
& =v_{(1)} w_{(\underline{1})} S\left(v_{(2)} w_{(2)}\right) \otimes_{H} v_{(\underline{3})} w_{(3)} \\
& =v_{(1)} w_{(\underline{1})} S w_{(2)} \otimes_{H} S v_{(2)} v_{(\underline{3})} w_{(3)} \\
& =\Psi \circ c_{\chi}\left(v \otimes_{H_{\chi}} w\right)
\end{aligned}
$$

We wrote $\chi^{\prime \prime}$ for a third copy of $\chi$ and used the notation

$$
\begin{gathered}
\chi^{(1)} \otimes \chi^{(2)} \otimes \chi^{(3)}=(1 \otimes \chi) \cdot(\mathrm{id} \otimes \Delta) \chi, \\
\chi^{-(1)} \otimes \chi^{-(2)} \otimes \chi^{-(3)}=(\Delta \otimes \mathrm{id}) \chi^{-1} \cdot\left(\chi^{-1} \otimes 1\right) .
\end{gathered}
$$


For the invertibility, it is clear from the formulae that twisting by $\chi^{-1}$ after twisting by $\chi$ gives the original objects and morphisms.

Next we give a different set of results where the product rather than the coproduct of $H$ is twisted. Note that the category of bicovariant bimodules over $H$ does not depend symmetrically on the product and coproduct of $H$ (for example, the tensor product in the category is $\otimes_{H}$ ). Hence the following theorem is not in any simple way the dual of the one above. We start with the corresponding lemma.

Lemma 2.4 Let $\chi: H \otimes H \rightarrow k$ be a unital 2-cocycle on $H$. Then $\chi$ extends to a graded unital 2-cocycle $\chi: T_{q} \Omega \otimes T_{q} \Omega \rightarrow k$ by defining $\chi$ to be zero on elements of degree $\geq 1$. Moreover, the twisted product yields $\left(T_{q} \Omega\right)^{\chi}$ as a bicovariant bimodule over $H^{\chi}$ and a $\mathbb{Z}_{q}$-braided group. In particular, the degree 0 part of $\left(T_{q} \Omega\right)^{\chi}$ is $H^{\chi}$.

Proof We observe that the counit vanishes on elements of degree $\geq 1$. The conditions for $\chi$ to be a graded unital 2-cocycle on $T_{q} \Omega$ thus reduce to the the conditions for $\chi$ to be a unital 2-cocycle on $H$.

Theorem 2.5 Let $H$ be a Hopf algebra, $\chi: H \otimes H \rightarrow k$ a unital 2-cocycle. There is an isomorphism of braided categories $\mathcal{G}^{\chi}:{ }_{H}^{H} \mathcal{M}_{H}^{H} \rightarrow{ }_{H}^{H^{\chi}} \mathcal{M}_{H^{\chi}}^{H^{\chi}} . \mathcal{G}^{\chi}$ leaves the coactions unchanged and transforms the actions according to

$$
\begin{aligned}
& h \bullet v=\chi\left(h_{(1)} \otimes v_{(1)}\right) h_{(2)} v_{(\underline{2})} \chi^{-1}\left(h_{(3)} \otimes v_{(3)}\right), \\
& v \bullet h=\chi\left(v_{(1)} \otimes h_{(1)}\right) v_{(\underline{2})} h_{(2)} \chi^{-1}\left(v_{(3)} \otimes h_{(3)}\right) .
\end{aligned}
$$

The monoidal structure is provided by the natural transformation

$$
\begin{aligned}
c^{\chi}: \mathcal{G}^{\chi}(V) \otimes_{H^{\chi}} \mathcal{G}^{\chi}(W) & \rightarrow \mathcal{G}^{\chi}\left(V \otimes_{H} W\right) \\
v \otimes_{H^{\chi}} w & \mapsto \chi\left(v_{(1)} \otimes w_{(1)}\right) v_{(\underline{2})} \otimes_{H} w_{(\underline{2})} \chi^{-1}\left(v_{(3)} \otimes w_{(3)}\right)
\end{aligned}
$$

for all $V, W \in{ }_{H}^{H} \mathcal{M}_{H}^{H}$.

Proof By restricting Lemma 2.4 to degree 1 we already know that $\mathcal{G}^{\chi}$ maps bicovariant bimodules to bicovariant bimodules. To see that $\mathcal{G}^{\chi}$ is indeed a functor we have to show that it maps morphisms to morphisms. Let $f: V \rightarrow W$ be a morphism in ${ }_{H}^{H} \mathcal{M}_{H}^{H}$. Since $\mathcal{G}^{\chi}$ leaves the coactions invariant, we just have to check that $f$ remains a module map. For all $h \in H, v \in V$,

$$
\begin{aligned}
f(h \bullet v) & =\chi\left(h_{(1)} \otimes v_{(1)}\right) f\left(h_{(2)} v_{(\underline{2})}\right) \chi\left(h_{(3)} \otimes v_{(3)}\right) \\
& =\chi\left(h_{(1)} \otimes v_{(1)}\right) h_{(2)} f\left(v_{(\underline{2})}\right) \chi\left(h_{(3)} \otimes v_{(3)}\right) \\
& =\chi\left(h_{(1)} \otimes(f(v))_{(1)}\right) h_{(2)}(f(v))_{(\underline{2})} \chi\left(h_{(3)} \otimes(f(v))_{(3)}\right) \\
& =h \bullet f(v) .
\end{aligned}
$$

Similarly for the right action.

Next, we show that $\mathcal{G}^{\chi}$ is monoidal. The associativity property of $c^{\chi}$ and invertibility follow from $\chi$ an invertible cocycle. We verify that $c^{\chi}$ is a morphism in ${ }_{H^{\chi}}^{H^{\chi}} \mathcal{M}_{H^{\chi}}^{H^{\chi}}$. As before, we only 
do the proof for the left action and left coaction; the right handed versions follow by symmetry. For the left coaction this is the commutativity of the diagram

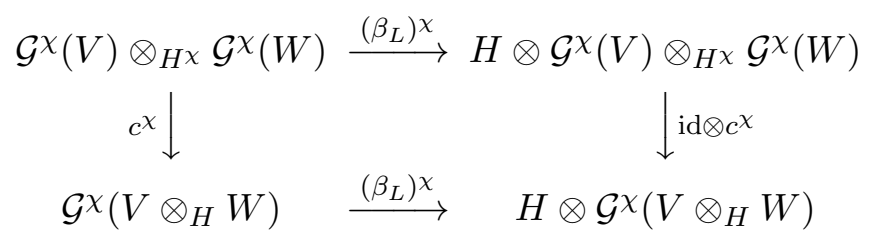

Explicitly,

$$
\begin{aligned}
\left(\beta_{L}\right)^{\chi} \circ c^{\chi}\left(v \otimes_{H} \chi w\right)= & \left(\beta_{L}\right)^{\chi}\left(\chi\left(v_{(1)} \otimes w_{(1)}\right) v_{(\underline{2})} \otimes_{H} w_{(\underline{2})} \chi^{-1}\left(v_{(3)} \otimes w_{(3)}\right)\right) \\
= & \chi\left(v_{(1)} \otimes w_{(1)}\right) v_{(2)} w_{(2)} \otimes v_{(\underline{3})} \otimes_{H} w_{(\underline{3})} \chi^{-1}\left(v_{(4)} \otimes w_{(4)}\right) \\
= & \chi\left(v_{(1)} \otimes w_{(1)}\right) v_{(2)} w_{(2)} \chi^{-1}\left(v_{(3)} \otimes w_{(3)}\right) \\
& \otimes \chi\left(v_{(4)} \otimes w_{(4)}\right) v_{(\underline{5})} \otimes_{H} w_{(\underline{5})} \chi^{-1}\left(v_{(6)} \otimes w_{(6)}\right) \\
= & \left(\operatorname{id} \otimes c^{\chi}\right)\left(v_{(1)} \bullet w_{(1)} \otimes v_{(\underline{2})} \otimes_{H \chi} w_{(\underline{2})}\right) \\
= & \left(\mathrm{id} \otimes c^{\chi}\right) \circ\left(\beta_{L}\right)^{\chi}\left(v \otimes_{H^{\chi}} w\right) .
\end{aligned}
$$

For the left action we require the commutativity of the diagram

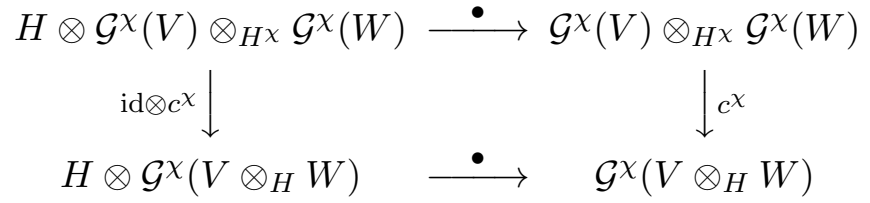

This is,

$$
\begin{aligned}
c^{\chi}\left(h \bullet\left(v \otimes_{H} \chi w\right)=\right. & c^{\chi}\left(h \bullet v \otimes_{H \chi} w\right) \\
= & \chi\left(h_{(1)} \otimes v_{(1)}\right) c^{\chi}\left(h_{(2)} v_{(\underline{2})} \otimes_{H} \chi w\right) \chi^{-1}\left(h_{(3)} \otimes v_{(3)}\right) \\
= & \chi\left(h_{(1)} \otimes v_{(1)}\right) \chi\left(h_{(2)} v_{(2)} \otimes w_{(1)}\right) h_{(3)} v_{(\underline{3})} \otimes_{H} w_{(\underline{2})} \\
& \chi^{-1}\left(h_{(4)} v_{(4)} \otimes w_{(3)}\right) \chi^{-1}\left(h_{(5)} \otimes v_{(5)}\right) \\
= & \chi\left(h_{(1)} \otimes v_{(1)}\right) \chi^{-1}\left(h_{(2)(1)} \otimes v_{(2)(1)}\right) \\
& \chi\left(v_{(2)(2)} \otimes w_{(1)(1)}\right) \chi\left(h_{(2)(2)} \otimes v_{(2)(3)} w_{(1)(2)}\right) h_{(3)} v_{(\underline{3})} \otimes_{H} w_{(\underline{2})} \\
& \chi^{-1}\left(h_{(4)} v_{(4)} \otimes w_{(3)}\right) \chi^{-1}\left(h_{(5)} \otimes v_{(5)}\right) \\
= & \chi\left(v_{(1)} \otimes w_{(1)}\right) \chi\left(h_{(1)} \otimes v_{(2)} w_{(2)}\right) h_{(2)} v_{(\underline{3})} \otimes_{H} w_{(\underline{3})} \\
& \chi^{-1}\left(h_{(3)} v_{(4)} \otimes w_{(4)}\right) \chi^{-1}\left(h_{(4)} \otimes v_{(5)}\right) \\
= & \chi\left(v_{(1)} \otimes w_{(1)}\right) \chi\left(h_{(1)} \otimes v_{(2)} w_{(2)}\right) h_{(2)} v_{(\underline{3})} \otimes_{H} w_{(\underline{3})} \\
& \chi^{-1}\left(h_{(3)(1)} \otimes v_{(4)(1)} w_{(4)(1)}\right) \chi^{-1}\left(v_{(4)(2)} \otimes w_{(4)(2)}\right) \\
& \chi\left(h_{(3)(2)} \otimes v_{(4)(3)}\right) \chi^{-1}\left(h_{(4)} \otimes v_{(5)}\right) \\
= & \chi\left(v_{(1)} \otimes w_{(1)}\right) \chi\left(h_{(1)} \otimes v_{(2)} w_{(2)}\right) h_{(2)} v_{(\underline{3})} \otimes_{H} w_{(\underline{3})} \\
& \chi^{-1}\left(h_{(3)} \otimes v_{(4)} w_{(4)}\right) \chi^{-1}\left(v_{(5)} \otimes w_{(5)}\right) \\
= & \chi\left(v_{(1)} \otimes w_{(1)}\right) \chi\left(h_{(1)} \otimes\left(v_{(\underline{2})} \otimes_{H} w_{(\underline{2})}\right)_{(1)}\right) h_{(2)}\left(v_{(\underline{2})} \otimes_{H} w_{(\underline{2})}\right)_{(\underline{2})} \\
& \chi^{-1}\left(h_{(3)} \otimes\left(v_{(\underline{2})} \otimes_{H} w_{(\underline{2})}\right)_{(3)}\right) \chi^{-1}\left(v_{(3)} \otimes w_{(3)}\right) \\
= & h \bullet\left(\chi\left(v_{(1)} \otimes w_{(1)}\right) v_{(\underline{2})} \otimes_{H} w_{(\underline{2})} \chi^{-1}\left(v_{(3)} \otimes w_{(3)}\right)\right) \\
= & h \bullet c^{\chi}\left(v \otimes_{H \chi} w\right) .
\end{aligned}
$$


Next we show that $\mathcal{G}^{\chi}$ preserves the braiding. This is the commutativity of the diagram

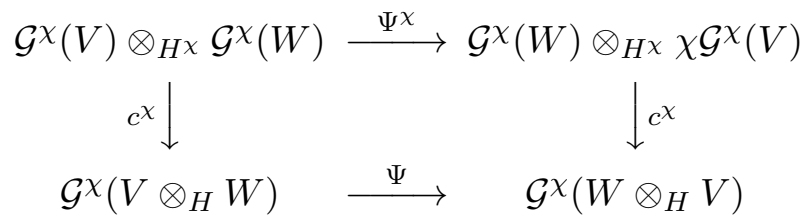

The braiding is $\Psi\left(h v \otimes_{H} w g\right)=h w \otimes_{H} v g$ with $h, g \in H, v \in V$ left-invariant and $w \in W$ right-invariant (similarly over $H^{\chi}$ ). Thus we have,

$$
\begin{aligned}
c^{\chi} \circ \Psi^{\chi}\left(h \bullet v \otimes_{H \chi} w \bullet g\right)= & c^{\chi}\left(h \bullet w \otimes_{H \chi} v \bullet g\right) \\
= & c^{\chi}\left(\chi\left(h_{(1)} \otimes w_{(1)}\right) h_{(2)} w_{(\underline{2})} \otimes_{H \chi} v_{(\underline{1})} g_{(1)} \chi^{-1}\left(v_{(2)} \otimes g_{(2)}\right)\right) \\
= & \chi\left(h_{(1)} \otimes w_{(1)}\right) \chi\left(h_{(2)} w_{(2)} \otimes g_{(1)}\right) h_{(3)} w_{(\underline{3})} \otimes_{H} v_{(\underline{1})} g_{(2)} \\
& \chi^{-1}\left(h_{(4)} \otimes v_{(2)} g_{(3)}\right) \chi^{-1}\left(v_{(3)} \otimes g_{(4)}\right) \\
= & \Psi\left(\chi\left(h_{(1)} \otimes w_{(1)}\right) \chi\left(h_{(2)} w_{(2)} \otimes g_{(1)}\right) h_{(3)} v_{(\underline{1})} \otimes_{H} w_{(\underline{3})} g_{(2)}\right. \\
& \left.\chi^{-1}\left(h_{(4)} \otimes v_{(2)} g_{(3)}\right) \chi^{-1}\left(v_{(3)} \otimes g_{(4)}\right)\right) \\
= & \Psi\left(\chi\left(w_{(1)} \otimes g_{(1)}\right) \chi\left(h_{(1)} \otimes w_{(2)} g_{(2)}\right) h_{(2)} v_{(\underline{1})} \otimes_{H} w_{(\underline{3})} g_{(3)}\right. \\
& \left.\chi^{-1}\left(h_{(3)} \otimes v_{(2)} g_{(4)}\right) \chi^{-1}\left(v_{(3)} \otimes g_{(5)}\right)\right) \\
= & \Psi\left(\chi\left(w_{(1)} \otimes g_{(1)}\right) \chi\left(h_{(1)} \otimes w_{(2)} g_{(2)}\right) h_{(2)} v_{(\underline{1})} \otimes_{H} w_{(\underline{3})} g_{(3)}\right. \\
& \left.\chi^{-1}\left(h_{(3)} v_{(2)} \otimes g_{(4)}\right) \chi^{-1}\left(h_{(4)} \otimes v_{(3)}\right)\right) \\
= & \Psi \circ c^{\chi}\left(\chi\left(w_{(1)} \otimes g_{(1)}\right) h_{(1)} v_{(\underline{1})} \otimes_{H \chi} w_{(\underline{2})} g_{(2)} \chi^{-1}\left(h_{(2)} \otimes v_{(2)}\right)\right) \\
= & \Psi \circ c^{\chi}\left(h \bullet v \otimes_{H \chi} w \bullet g .\right.
\end{aligned}
$$

Only the invertibility remains to be shown. The inverse operation to twisting by $\chi$ is twisting by $\chi^{-1}$. Since both, the twisting of the actions and the natural transformation $c^{\chi}$ look formally exactly like the twisting of the product in $H$, we see that applying $\chi^{-1}$ after $\chi$ will give the original object in the same way as for the product in $H$.

From these results we obtain in particular the twisting of the bicovariant bimodule $\Omega$ itself to $\Omega_{\chi}$ and $\Omega^{\chi}$ in the two cases. Finally, we are able to identify the braided groups in the above lemmas,

Corollary $2.6\left(T_{q} \Omega\right)_{\chi}=T_{q}\left(\Omega_{\chi}\right)$ and $\left(T_{q} \Omega\right)^{\chi} \cong T_{q}\left(\Omega^{\chi}\right)$ in the settings of Theorem 2.3 and 2.5 respectively.

Proof Given a bicovariant bimodule $\Omega$ over a Hopf algebra $H$ we can identify $\left(T_{q} \Omega\right)_{\chi}$ with $\left(T_{q} \Omega_{\chi}\right)$ using the natural transformation $c_{\chi}$ in Theorem 2.3 extended to multiple tensor products. Similarly for ()$^{\chi}$ using Theorem 2.5. Since $c$ preserves degree, this identification is graded (a morphism in the category of $\mathbb{Z}_{q}$-comodules).

\subsection{Twisting of crossed modules}

In this section we restrict the above results to the right-invariant part of the bicovariant bimodule $\Omega$. This is a crossed module $V$ and $\Omega=V \otimes H$ as explained in the preliminaries. In this way we obtain, as corollaries of the preceeding subsection, results about the response of crossed modules under twisting. This is relevant to the our treatment of differential calculi but it is also of independent interest in several other algebraic settings where crossed modules play an important role. For this reason some more direct proofs are provided in the appendix. 
Theorem 2.7 Let $H$ be a Hopf algebra, $\chi: H \otimes H \rightarrow k$ a unital 2-cocycle. There is an isomorphism of braided categories $\mathcal{F} \chi:{ }_{H}^{H} \dot{\mathcal{M}} \rightarrow{ }_{H}^{H} \chi \dot{\mathcal{M}}$ given by the identity on the underlying vector spaces and coactions, and transforming the action $\triangleright$ to

$$
h \triangleright^{\chi} v=\chi\left(h_{(1)} \otimes v_{(1)}\right)\left(h_{(2)} \triangleright v_{(\infty)}\right)_{(\infty)} \chi^{-1}\left(\left(h_{(2)} \triangleright v_{(\infty)}\right)_{(1)} \otimes h_{(3)}\right) .
$$

The monoidal structure is given by the natural transformation

$$
c^{\chi}: \mathcal{F}^{\chi}(V) \otimes \mathcal{F}^{\chi}(W) \rightarrow \mathcal{F}^{\chi}(V \otimes W), \quad v \otimes w \mapsto \chi\left(v_{(1)} \otimes w_{(1)}\right) v_{(\infty)} \otimes w_{(\infty)} .
$$

Proof We deduce this from Theorem 2.5 using the equivalence of categories ${ }_{H}^{H} \mathcal{M}_{H}^{H} \cong{ }_{H}^{H} \dot{\mathcal{M}}$. As explained in the Preliminaries, a bicovariant bimodule has the canonical form $\Omega=V \otimes H$ with $V$ a crossed module. Conversely, $V$ may be recovered as the space of right-invariant forms of $\Omega$ with the action $\triangleright$ recovered from the bimodule structure on $\Omega$ (denoted ·) by

$$
h \triangleright v=h_{(1)} \cdot v \cdot S h_{(2)} .
$$

Since the twisting in ${ }_{H}^{H} \mathcal{M}_{H}^{H}$ preserves the coactions, it preserves the decomposition $\Omega=V \otimes H$ and thus induces a twisting ${ }_{H}^{H} \dot{\mathcal{M}} \rightarrow{ }_{H \chi}^{H^{\chi}} \dot{\mathcal{M}}$ by restriction to the right-invariant forms $V$. The coaction of the crossed module remains unchanged, while the twisted action in ${ }_{H \chi}^{H^{\chi}} \dot{\mathcal{M}}$ becomes (we denote the twisted actions by $\triangleright^{\chi}$ and $\bullet$ respectively):

$$
\begin{aligned}
h \triangleright^{\chi} v & =h_{(1)} \bullet v \bullet S^{\chi} h_{(2)} \\
& =h_{(1)} \bullet v \bullet S h_{(3)} U\left(h_{(2)}\right) U^{-1}\left(h_{(4)}\right) \\
& =\chi\left(h_{(1)} \otimes v_{(1)}\right) h_{(2)} \cdot v_{(\underline{2})} \bullet S h_{(4)} U\left(h_{(3)}\right) U^{-1}\left(h_{(5)}\right) \\
& =\chi\left(h_{(1)} \otimes v_{(1)}\right) \chi\left(h_{(2)} v_{(2)} \otimes S h_{(8)}\right) h_{(3)} \cdot v_{(\underline{3})} \cdot S h_{(7)} \chi^{-1}\left(h_{(4)} \otimes S h_{(6)}\right) U\left(h_{(5)}\right) U^{-1}\left(h_{(9)}\right) \\
& =\chi\left(h_{(1)} \otimes v_{(1)}\right) \chi\left(h_{(2)} v_{(2)} \otimes S h_{(5)}\right) h_{(3)} \cdot v_{(\underline{3})} \cdot S h_{(4)} U^{-1}\left(h_{(6)}\right) \\
& =\chi\left(h_{(1)} \otimes v_{(1)}\right) \chi\left(h_{(2)} v_{(2)} \otimes S h_{(4)}\right) h_{(3)} \triangleright v_{(\underline{3})} U^{-1}\left(h_{(5)}\right) \\
& =\chi\left(h_{(1)} \otimes v_{(1)}\right) \chi\left(\left(h_{(2)} \triangleright v_{(\underline{2})}\right)_{(1)} h_{(3)} \otimes S h_{(4)}\right)\left(h_{(2)} \triangleright v_{(\underline{2})}\right)_{(\underline{2})} U^{-1}\left(h_{(5)}\right) \\
& =\chi\left(h_{(1)} \otimes v_{(1)}\right)\left(h_{(2)} \triangleright v_{(\underline{2})}\right)_{(\underline{2})} \chi^{-1}\left(\left(h_{(2)} \triangleright v_{(\underline{2})}\right)_{(1)} \otimes h_{(3)}\right) .
\end{aligned}
$$

We used the identity $\chi\left(a h_{(1)} \otimes S h_{(2)}\right) U^{-1}\left(h_{(3)}\right)=\chi^{-1}(a \otimes h)$ and convert to a more conventional comodule notation as stated. Finally, for the monoidal structure, restricting $c^{\chi}$ given in Theorem 2.5 to the right-invariant component leads to the stated form. The fact that $\mathcal{F}^{\chi}$ preserves the braiding just follows from the fact that the braiding in ${ }_{H}^{H} \dot{\mathcal{M}}$ is induced by the braiding in ${ }_{H}^{H} \mathcal{M}_{H}^{H}$. The isomorphism property follows from the categorial equivalence ${ }_{H}^{H} \mathcal{M}_{H}^{H} \cong{ }_{H}^{H} \dot{\mathcal{M}}$.

We proceed to give the dual version of this theorem. Note that it can not be derived from Theorem 2.3 in a way analogous to the above proof. The reason is essentially that Theorems 2.5 and 2.3 are not strictly dual to each other. This is because the tensor product $\otimes_{H}$ is not self-dual in our sense. Dualisation instead converts this tensor product to the corresponding cotensor product. Since we do not want to concern ourselves with that here, we give a proof by dualisation of Theorem 2.7. In the crossed module setting this presents no further problem since the tensor product in ${ }_{H}^{H} \dot{\mathcal{M}}$ is the usual one and thus self-dual in our sense.

Theorem 2.8 Let $H$ be a Hopf algebra, $\chi \in H \otimes H$ a counital 2-cocycle for $H$. There is an isomorphism of braided categories $\mathcal{F}_{\chi}:{ }_{H}^{H} \dot{\mathcal{M}} \rightarrow{ }_{H_{\chi}}^{H_{\chi}} \dot{\mathcal{M}}$ given by the identity on the underlying vector spaces and actions, and transforming the coaction $\beta(v)=v_{(1)} \otimes v_{(\infty)}$ to

$$
\beta_{\chi}(v)=\chi^{(1)}\left(\chi^{-(1)} \triangleright v\right)_{(1)} \chi^{-(2)} \otimes \chi^{(2)} \triangleright\left(\chi^{-(1)} \triangleright v\right)_{(\infty)} .
$$


The monoidal structure is given by the natural transformation

$$
c_{\chi}: \mathcal{F}_{\chi}(V) \otimes \mathcal{F}_{\chi}(W) \rightarrow \mathcal{F}_{\chi}(V \otimes W), \quad v \otimes w \mapsto \chi^{-(1)} \triangleright v \otimes \chi^{-(2)} \triangleright w .
$$

Proof This Theorem is strictly dual to Theorem 2.7 and therefore equivalent: We can write the action of Theorem 2.7 as

$$
\begin{aligned}
\triangleright^{\chi}= & \left(\chi^{-1} \otimes \mathrm{id}\right) \circ(\mathrm{id} \otimes \tau) \circ(\beta \otimes \mathrm{id}) \circ(\triangleright \otimes \mathrm{id}) \circ(\mathrm{id} \otimes \tau) \\
& \circ(\Delta \otimes \mathrm{id}) \circ(\chi \otimes \mathrm{id} \otimes \mathrm{id}) \circ(\mathrm{id} \otimes \tau \otimes \mathrm{id}) \circ(\Delta \otimes \beta)
\end{aligned}
$$

with $\chi: H \otimes H \rightarrow k$ and $\tau$ the flip map. Dualising means reversing the order of the composition, exchanging product with coproduct, action with coaction and switching to $\chi: k \rightarrow H \otimes H$ :

$$
\begin{aligned}
\beta_{\chi}= & (\cdot \otimes \triangleright) \circ(\mathrm{id} \otimes \tau \otimes \mathrm{id}) \circ(\chi \otimes \mathrm{id} \otimes \mathrm{id}) \circ(\cdot \otimes \mathrm{id}) \\
& \circ(\mathrm{id} \otimes \tau) \circ(\beta \otimes \mathrm{id}) \circ(\triangleright \otimes \mathrm{id}) \circ(\mathrm{id} \otimes \tau) \circ\left(\chi^{-1} \otimes \mathrm{id}\right) .
\end{aligned}
$$

This is just the coaction stated. For the monoidal structure, we have to take into account that $c$ flips its direction under dualisation. So the dual of

$$
c^{\chi}=(\chi \otimes \mathrm{id} \otimes \mathrm{id}) \circ(\mathrm{id} \otimes \tau \otimes \mathrm{id}) \circ(\beta \otimes \beta)
$$

in Theorem 2.7 is

$$
\left(c_{\chi}\right)^{-1}=(\triangleright \otimes \triangleright) \circ(\mathrm{id} \otimes \tau \otimes \mathrm{id}) \circ(\chi \otimes \mathrm{id} \otimes \mathrm{id}) .
$$

Inverting replaces $\chi^{-1}$ with $\chi$, leading to the formula stated.

Corollary 2.9 Let $H$ be finite-dimensional and $D(H)$ its quantum double. Then there is a Hopf algebra isomorphism $\theta: D\left(H^{\chi}\right) \cong D(H)_{\tilde{\chi}}$ where $\tilde{\chi}=\chi^{-1}$ viewed in $D(H) \otimes D(H)$, and the twisting is that of the coproduct of $D(H)$. Here,

$$
\theta(\phi \otimes h)=\chi_{(1)}^{-(2)} \otimes \chi^{\prime-(1)} \phi \otimes h_{(2)}\left\langle h_{(1)}, U S \chi^{-(1)}\right\rangle\left\langle h_{(3)}, \chi_{(2)}^{-(2)} \chi^{\prime-(2)}\right\rangle, \quad \forall \phi \in H^{*}, h \in H .
$$

Proof This follows by Tannaka-Krein arguments [5]. Thus, there is a uniquely determined algebra isomorphism $\theta$ such that $\mathcal{F}^{\chi}$ is pull-back along $\theta$, and this is a bialgebra map up to a conjugation corresponding to the nontrivial isomorphism of tensor products of objects in the image of $\mathcal{F} \chi$ in Theorem 2.7. We build $D(H)$ explicitly on $H^{*} \otimes H$ as explained in the Preliminaries. Then,

$$
\begin{aligned}
\theta(\phi \otimes h) \triangleright v & =(\phi \otimes h) \triangleright \chi \\
& \left.=\phi\left(\left(h_{(2)} \triangleright v_{(\infty)}\right)_{(\infty)(1)}\right)\left(h_{(2)} \triangleright v_{(\infty)}\right)_{(\infty)(\infty)} \chi\left(h_{(1)} \otimes v_{(1)}\right) \chi^{-1}\left(\left(h_{(2)} \triangleright v_{(\infty)}\right)_{(1)} \otimes h_{(3)}\right)\right) \\
& \left.=\phi\left(\left(h_{(2)} \triangleright v_{(\infty)}\right)_{(1)(2)}\right)\left(h_{(2)} \triangleright v_{(\infty)}\right)_{(\infty)} \chi\left(h_{(1)} \otimes v_{(1)}\right) \chi^{-1}\left(\left(h_{(2)} \triangleright v_{(\infty)}\right)_{(1)} \otimes h_{(3)}\right)\right) \\
& =\phi \cdot \chi^{-(1)} \triangleright\left(h_{(2)} \triangleright v_{(\infty)}\right)\left\langle\chi^{-(2)}, h_{(3)}\right\rangle \chi\left(h_{(1)} \otimes v_{(1)}\right) \\
& =\phi \cdot \chi^{-(1)} \cdot h_{(2)} \cdot \chi^{(2)} \triangleright v\left\langle\chi^{-(2)}, h_{(3)}\right\rangle\left\langle\chi^{(1)}, h_{(1)}\right\rangle \\
& =\phi \cdot \chi^{-(1)} \cdot \chi_{(2)}^{(2)} \cdot h_{(2)(2)} \triangleright v\left\langle\chi^{-(2)}, h_{(3)}\right\rangle\left\langle\chi^{(1)}, h_{(1)}\right\rangle\left\langle S \chi_{(2)}^{(2)}, h_{(2)(1)}\right\rangle\left\langle\chi_{(3)}^{(2)}, h_{(2)(3)}\right\rangle \\
& =\phi \cdot \chi^{-(1)} \cdot \chi_{(2)}^{(2)} \cdot h_{(2)} \triangleright v\left\langle h_{(1)}, \chi^{(1)} S \chi_{(1)}^{(2)}\right\rangle\left\langle h_{(3)}, \chi_{(3)}^{(2)} \chi^{-(2)}\right\rangle
\end{aligned}
$$


using the definition of $\triangleright^{\chi}$, the definition of the action of $D(H)$ on $v \in V$ (namely $\phi$ acts by evaluation against the coaction), then using the cross relations of $D(H)$ and the duality pairing axioms. Here $\cdot$ denotes the product in $D(H)$. From this and from the cocycle identity

$$
\chi^{(1)} S \chi_{(1)}^{(2)} \otimes \chi_{(2)}^{(2)}=\chi^{(1)}\left(S \chi^{(2)}\right) S \chi^{-(1)} \otimes \chi^{-(2)}
$$

(which follows from id $\otimes S$ and the product applied to (id $\otimes$ id) $\chi=\chi_{23}^{-1} \chi_{12}(\Delta \otimes$ id) $\chi$ ), we find that

$$
\theta(\phi \otimes h)=\chi_{(2)}^{(2)} \chi^{-(1)} \phi \otimes h_{(2)} \triangleright v\left\langle h_{(1)}, \chi^{(1)} S \chi_{(1)}^{(2)}\right\rangle\left\langle h_{(3)}, \chi_{(3)}^{(2)} \chi^{-(2)}\right\rangle
$$

(where the products are in $H^{*}$ and $H$ ) has the form stated. Next, we note that if two Hopf algebras have their coproducts related by twisting by $\chi$ then the induced monoidal functor has the same form as for $\mathcal{F}^{\chi}$ on tensor products [5]. From this, we conclude that $\theta$ is an isomorphism to $D(H)_{\tilde{\chi}}$ where $\tilde{\chi}=\chi^{-1} \in H^{*} \otimes H^{*}$ is viewed in $D(H) \otimes D(H)$. Since $H^{* o p} \subseteq D(H)$ as a subalgebra, conjugation by $\chi$ using the product of $H^{*}$ is actually conjugation by $\chi^{-1}$ in $D(H)$. That $\theta$ is then an isomorphism of bialgebras can also be checked explicitly.

Clearly, the dual of the double $D(H)^{*}$ changes to $D(H)^{* \tilde{\chi}}$ similarly. For completeness, let us mention also the dual version of Corollary 2.9. Let $\chi \in H \otimes H$ be a cocycle and $H_{\chi}$ the Hopf algebra with twisted coproduct. Since the double $D(H)$ involves both $H$ and $H^{* o p}$ equally, $D\left(H_{\chi}\right)$ is likewise a twisting.

\section{Twisting of differential calculi and exterior algebras}

In this section we apply the technical results of Section 2 to obtain our main result, which is a twisting theory for first order and exterior differential calculi. We fix a Hopf algebra $H$ and a first order differential calculus $\left(\Omega^{1}, \mathrm{~d}\right)$. We begin with a more explicit discussion of the maximal prolongation exterior algebra that we have found elsewhere, and the analogue of Brzezinski's theorem [15] for it. We will then study how it, and the more well-known Woronowicz exterior algebra, respond under twisting.

Proposition 3.1 The maximal prolongation $\widetilde{\Omega}=\oplus_{n} \Omega^{n}$ is a quotient of $T_{-1} \Omega^{1}$ by the ideal generated by

$$
I=\left\{\mathrm{d} a_{i} \otimes \mathrm{d} b_{i} \mid a_{i} \mathrm{~d} b_{i}=0\right\} \subseteq \Omega_{H}^{1} \underset{H}{\otimes} \Omega^{1} .
$$

Here $\mathrm{d}$ extends to $\mathrm{d}: \widetilde{\Omega} \rightarrow \widetilde{\Omega} \cdot$ by $\mathrm{d}^{2}=0$ and the graded Leibniz rule

$$
\mathrm{d}(\alpha \underset{H}{\otimes} \beta)=\mathrm{d} \alpha \underset{H}{\otimes} \beta+(-1)^{|\alpha|} \alpha \underset{H}{\otimes} \mathrm{d} \beta
$$

for $\alpha$ of degree $|\alpha|$. Moreover, $\widetilde{\Omega} \cdot$ remains a super-Hopf algebra and $\mathrm{d}$ commutes with its coproduct and antipode. 
Proof We first observe that $I$ is itself a bicovariant bimodule. For the coactions this follows from the fact that $\mathrm{d}$ is a bicomodule map. For the actions suppose $a_{i} \mathrm{~d} b_{i}=0$. Then

$$
c \mathrm{~d} a_{i} \underset{H}{\otimes} \mathrm{d} b_{i}=\mathrm{d}\left(c a_{i}\right) \underset{H}{\otimes} \mathrm{d} b_{i}-\mathrm{d} c \underset{H}{\otimes} a_{i} \mathrm{~d} b_{i}=\mathrm{d}\left(c a_{i}\right) \underset{H}{\otimes} \mathrm{d} b_{i} \in I
$$

for all $c \in H$, since $c a_{i} \mathrm{~d} b_{i}=0$. On the other side

$$
\mathrm{d} a_{i} \underset{H}{\otimes}\left(\mathrm{d} b_{i}\right) c=\mathrm{d} a_{i} \underset{H}{\otimes} \mathrm{d}\left(b_{i} c\right)-\mathrm{d} a_{i} \underset{H}{\otimes} b_{i} \mathrm{~d} c=\mathrm{d} a_{i} \underset{H}{\otimes} \mathrm{d}\left(b_{i} c\right)-\mathrm{d}\left(a_{i} b_{i}\right) \underset{H}{\otimes} \mathrm{d} c+a_{i} \mathrm{~d} b_{i} \underset{H}{\otimes} \mathrm{d} c \in I
$$

since $a_{i} \mathrm{~d}\left(b_{i} c\right)-a_{i} b_{i} \mathrm{~d} c=a_{i}\left(\mathrm{~d} b_{i}\right) c=0$. We then define $\mathrm{d}: \Omega^{1} \rightarrow \Omega^{1} \otimes_{H} \Omega^{1} / I=\Omega^{2}$ by $\mathrm{d}(a \mathrm{~d} b)=$ $\mathrm{d} a \otimes_{H} \mathrm{~d} b$. This is well-defined in virtue of the definition of $I$ and $\mathrm{d}^{2}=0$, and also obeys the Leibniz rule $\mathrm{d}(a \alpha)=\mathrm{d} a \otimes_{H} \alpha+a \mathrm{~d} \alpha$ (and similarly with $a$ on the other side). We then extend to products of degree 1 forms by the braided-Leibniz rule as stated. It is well-defined on $\alpha \otimes_{H} \beta$ in view of the above Leibniz rule with $a \in H$ and moreover itself obeys this Leibniz rule with $\alpha$ now of higher degree. It maps to $\Omega^{3}=\Omega^{2} \otimes_{H} \Omega^{1}+\Omega^{1} \otimes_{H} \Omega^{2}$. In this way one constructs $\Omega^{n}$ and $\mathrm{d}$ by induction. In all higher degrees $\Omega^{n}$ is the quotient of $\Omega^{1} \otimes_{H} \cdots \otimes_{H} \Omega^{1}$ by $\sum \Omega^{1} \otimes_{H} \cdots \otimes_{H} I \otimes_{H} \cdots \otimes_{H} \Omega^{1}$ as stated. It is then straightforward to verify by induction that $\mathrm{d}^{2}=0$ to all orders. Next, we check that $\widetilde{\Omega}$ remains a super-Hopf algebra as a quotient of $T_{-1} \Omega^{1}$. There are general arguments for this, however in our case it is enough to verify that $\Delta I \subseteq I \otimes H+H \otimes I$ in $T_{-1} \Omega^{1}$. Thus,

$$
\begin{aligned}
\Delta\left(\mathrm{d} a_{i} \otimes_{H} \mathrm{~d} b_{i}\right)= & \left(\Delta \mathrm{d} a_{i}\right) \otimes_{H}\left(\Delta \mathrm{d} b_{i}\right) \\
= & \left(\mathrm{d} \Delta a_{i}\right) \otimes_{H}\left(\mathrm{~d} \Delta b_{i}\right) \\
= & \left(\mathrm{d} a_{i(1)} \otimes a_{i(2)}+a_{i(1)} \otimes \mathrm{d} a_{i(2)}\right) \otimes_{H}\left(\mathrm{~d} b_{i(1)} \otimes b_{i(2)}+b_{i(1)} \otimes \mathrm{d} b_{i(2)}\right) \\
= & \mathrm{d} a_{i(1)} \otimes_{H} \mathrm{~d} b_{i(1)} \otimes a_{i(2)} b_{i(2)}+\left(\mathrm{d} a_{i(1)}\right) b_{i(1)} \otimes a_{i(2)} \mathrm{d} b_{i(2)} \\
& -a_{i(1)} \mathrm{d} b_{i(1)} \otimes\left(\mathrm{d} a_{i(2)}\right) b_{i(2)}+a_{i(1)} b_{i(1)} \otimes \mathrm{d} a_{i(2)} \otimes_{H} \mathrm{~d} b_{i(2)} \\
= & \mathrm{d} a_{i(1)} \otimes_{H} \mathrm{~d} b_{i(1)} \otimes a_{i(2)} b_{i(2)}+\mathrm{d}\left(a_{i(1)} b_{i(1)}\right) \otimes a_{i(2)} \mathrm{d} b_{i(2)} \\
& -a_{i(1)} \mathrm{d} b_{i(1)} \otimes \mathrm{d}\left(a_{i(2)} b_{i(2)}\right)+a_{i(1)} b_{i(1)} \otimes \mathrm{d} a_{i(2)} \otimes_{H} \mathrm{~d} b_{i(2)} \\
= & \left(\mathrm{d} a_{i} \otimes_{H} \mathrm{~d} b_{i}\right)_{(0)} \otimes\left(\mathrm{d} a_{i} \otimes_{H} \mathrm{~d} b_{i}\right)_{(1)}+\mathrm{d}\left(a_{i} \mathrm{~d} b_{i}\right)_{(1)} \otimes\left(a_{i} \mathrm{~d} b_{i}\right)_{(\infty)} \\
& -\left(a_{i} \mathrm{~d} b_{i}\right)_{(0)} \otimes \mathrm{d}\left(a_{i} \mathrm{~d} b_{i}\right)_{(1)}+\left(\mathrm{d} a_{i} \otimes_{H} \mathrm{~d} b_{i}\right)_{(1)} \otimes\left(\mathrm{d} a_{i} \otimes_{H} \mathrm{~d} b_{i}\right)_{(\infty)} .
\end{aligned}
$$

While the second and third term are obviously zero, the first and the fourth term fulfil the condition since $I$ is a bicomodule. Similarly, one checks that $S I \subseteq I$. Finally, it is clear by induction that d commutes with $\Delta$ and $S$ in view of the first order d a bicomodule map. This step is the same as in 15] for the Woronowicz-type exterior algebras.

The maximal prolongation here is a quadratic algebra (over $H$ ) with relations $I$ in degree 2 . It is possible to generalise the construction to general $\widetilde{\Omega}_{q}$ similarly as a quotient of $T_{q} \Omega^{1}$, but it will no longer be quadratic. For example if $q$ is a primitive $n$-th root of unity. Thus, for $n=3$ one must specify

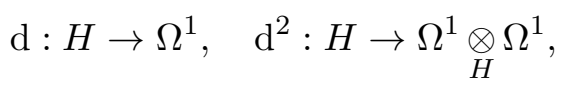

where $\mathrm{d}$ obeys the Leibniz rule (a given first order calculus) and $\mathrm{d}^{2}$ obeys the higher Leibniz rule

$$
\mathrm{d}^{2}(a b)=\left(\mathrm{d}^{2} a\right) b+[2]_{q} \mathrm{~d} a \underset{H}{\otimes} \mathrm{d} b+a \mathrm{~d}^{2} b
$$


with $[2]_{q}=1+q$. We then define $\mathrm{d}(a \mathrm{~d} b)=\mathrm{d} a \otimes_{H} \mathrm{~d} b+a \mathrm{~d}^{2} b$ with a modified definition

$$
I=\left\{\mathrm{d} a_{i} \underset{H}{\otimes} \mathrm{d} b_{i}+a_{i} \mathrm{~d}^{2} b_{i} \mid a_{i} \mathrm{~d} b_{i}=0\right\}
$$

in degree 2. Similarly, we define d on degree 2 and higher by the graded-Leibnitz rule with $q^{\text {। }}$ in place of $(-1)^{\mid} \mid$but must now quotient further in degree 3 for this to be well-defined (a cubic relation). By construction, $\mathrm{d}^{2}=\mathrm{d} \circ \mathrm{d}$ and $\mathrm{d}^{3}=0$. In this way one may build up a generalised complex in the spirit of [16].

One may similarly define a general exterior algebra $\Omega$ associated to a first order bicovariant calculus $\Omega^{1}$ as any super-Hopf algebra quotient of $T_{-1} \Omega^{1}$ in Proposition 2.1 with $H, \Omega^{1}$ in degrees 0,1 and such that d extends as a differential graded algebra and commutes with $\Delta, S$.

Proposition 3.2 Let $H$ be a Hopf algebra, $\chi: H \otimes H \rightarrow k$ a unital 2-cocycle. Then first order bicovariant differential calculi and exterior (super-Hopf) algebras over $H$ and $H^{\chi}$ are in one-to-one correspondence by the functor $\mathcal{G}^{\chi} . \mathcal{G}^{\chi}$ is trivial on $\mathrm{d}$.

Proof The degree 1 part as a bicovariant bimodule twists by the functor $\mathcal{G}^{\chi}$, as does the entire tensor super-Hopf algebra $T_{-1} \Omega^{1}$ in Corollary 2.6. By the same arguments (Lemma 2.4 and Theorem 2.5) this descends to any super-Hopf algebra quotient $\Omega^{\circ}$ of $T_{-1} \Omega^{1}$ and yields $\left(\Omega^{*}\right)^{\chi}$ as a super-Hopf algebra of the desired form. To see that this is compatible with the same $d$ requires us to check the Leibnitz rule. This is,

$$
\begin{aligned}
\mathrm{d}(\alpha \bullet \beta)= & \chi\left(\alpha_{(1)} \otimes \beta_{(1)}\right) \mathrm{d}\left(\alpha_{(\underline{2})} \beta_{(\underline{2})}\right) \chi^{-1}\left(\alpha_{(3)} \otimes \beta_{(3)}\right) \\
= & \chi\left(\alpha_{(1)} \otimes \beta_{(1)}\right)\left(\left(\mathrm{d} \alpha_{(\underline{2})}\right) \beta_{(\underline{2})}+(-1)^{\left|\alpha_{(\underline{2})}\right|} \alpha_{(\underline{2})} \mathrm{d} \beta_{(\underline{2})}\right) \chi^{-1}\left(\alpha_{(3)} \otimes \beta_{(3)}\right) \\
= & \chi\left((\mathrm{d} \alpha)_{(1)} \otimes \beta_{(1)}\right)(\mathrm{d} \alpha)_{(\underline{2})} \beta_{(\underline{2})} \chi^{-1}\left((\mathrm{~d} \alpha)_{(3)} \otimes \beta_{(3)}\right) \\
& +(-1)^{|\alpha|} \chi\left(\alpha_{(1)} \otimes(\mathrm{d} \beta)_{(1)}\right) \alpha_{(\underline{2})}(\mathrm{d} \beta)_{(\underline{2})} \chi^{-1}\left(\alpha_{(3)} \otimes(\mathrm{d} \beta)_{(3)}\right) \\
= & (\mathrm{d} \alpha) \bullet \beta+(-1)^{|\alpha|} \alpha \bullet \mathrm{d} \beta .
\end{aligned}
$$

Since the bicomodule and coproduct structure is unaffected by the twist, $d$ continues to be a bicomodule map and commute with the coaction (so in particular, $\left(\Omega^{1 \chi}, \mathrm{d}\right.$ ) is a first order calculus in $H^{\chi}$ ). Finally, the commutation of the antipode with $\mathrm{d}$ is

$$
\begin{aligned}
S^{\chi} \mathrm{d} \alpha & =U\left((\mathrm{~d} \alpha)_{(1)}\right) S\left((\mathrm{~d} \alpha)_{(\underline{2})}\right) U^{-1}\left((\mathrm{~d} \alpha)_{(3)}\right) \\
& =U\left(\alpha_{(1)}\right) S\left(\mathrm{~d} \alpha_{(2)}\right) U^{-1}\left(\alpha_{(3)}\right) \\
& =U\left(\alpha_{(1)}\right) \mathrm{d} S\left(\alpha_{(2)}\right) U^{-1}\left(\alpha_{(3)}\right) \\
& =\mathrm{d} S^{\chi} \alpha .
\end{aligned}
$$

This tells us that given an exterior algebra $\Omega$ on $H$, its twist by $\chi$ is some other exterior algebra $\Omega^{\chi \chi}$ on $H^{\chi}$.

Corollary 3.3 The maximal prolongation is stable under twisting, i.e. $(\widetilde{\Omega})^{\chi}$ is isomorphic via $c^{\chi}$ to the maximal prolongation of $\Omega^{1 \chi}$. 
Proof By Proposition 3.2, $(\widetilde{\Omega} \cdot)^{\chi}$ is an exterior algebra. Since $\widetilde{\Omega} \cdot$ is a quotient of $T_{-1} \Omega^{1}$, we just have to ensure that $c^{\chi}$, which identifies $\left(T_{-1} \Omega^{1}\right)^{\chi}$ with $T_{-1}\left(\Omega^{1 \chi}\right)$ according to Corollary 2.6 identifies the corresponding ideal $I$ of Proposition 3.1 with its twisted counterpart. This is

$$
\begin{aligned}
I^{\chi} & =\left\{\mathrm{d} a_{i} \otimes_{H \chi} \mathrm{d} b_{i} \mid a_{i} \bullet \mathrm{d} b_{i}=0\right\} \\
& =\left\{\mathrm{d} a_{i} \otimes_{H \chi} \mathrm{d} b_{i} \mid \chi\left(a_{i(1)} \otimes b_{i(1)}\right) a_{i(2)} \mathrm{d} b_{i(2)} \chi^{-1}\left(a_{i(3)} \otimes b_{i(3)}\right)=0\right\} \\
& =\left\{\chi^{-1}\left(a_{i(1)} \otimes b_{i(1)}\right) \mathrm{d} a_{i(2)} \otimes_{H \chi} \mathrm{d} b_{i(2)} \chi\left(a_{i(3)} \otimes b_{i(3)}\right) \mid a_{i} \mathrm{~d} b_{i}=0\right\} \\
& =c^{\chi}\left(\left\{\mathrm{d} a_{i} \otimes_{H} \mathrm{~d} b_{i} \mid a_{i} \mathrm{~d} b_{i}=0\right\}\right)=c^{\chi}(I) .
\end{aligned}
$$

The third equality here is by substitution of $\chi^{-1}\left(a_{i(1)} \otimes b_{i(1)}\right) a_{i(2)} \otimes b_{i(2)} \chi\left(a_{i(3)} \otimes b_{i(3)}\right)$ for $a_{i} \otimes b_{i}$.

We also have a similar result for the Woronowicz exterior algebra $\widehat{\Omega}$. As explained in the Preliminaries this is also a quotient of $T_{-1} \Omega^{1}$ but this time by skew-braid relations.

Corollary 3.4 The Woronowicz exterior algebra is stable under twisting, i.e. $\left(\widehat{\Omega}^{\cdot}\right)^{\chi}$ is isomorphic via $c^{\chi}$ to the Woronowicz construction based on $\Omega^{1 \chi}$.

Proof By Proposition 3.2, $(\widehat{\Omega})^{\chi}$ is an exterior algebra. Similarly to the preceding case, we view $\widehat{\Omega}$ as a quotient of $T_{-1} \Omega^{1}$ and have to ensure that $c^{\chi}$ is an intertwiner for the Woronowicz ideal by which we quotient. But this ideal is given as the kernel of a linear combination of identities and (bicovariant bimodule) braidings (see Preliminaries) and $c$ is an intertwiner for the braiding, so this is satisfied.

Finally, we have explained in the Preliminaries that bicovariant calculi are of the form $\Omega^{1}=$ $V \otimes H$ where $V$ is a quotient of $\operatorname{ker} \epsilon \in{ }_{H}^{H} \dot{\mathcal{M}}$. Here ker $\epsilon$ and $H$ itself are crossed modules by left multiplication and the left adjoint coaction. To complete our picture,

Proposition 3.5 We denote by $\pi: \operatorname{ker} \epsilon \rightarrow V$ the projection corresponding to $\Omega^{1}$ on $H$. Then the calculus $\Omega^{1 \chi}$ on $H^{\chi}$ corresponds to the projection $\pi^{\chi}=\pi \circ \alpha^{-1}$, where

$$
\alpha: \mathcal{F}^{\chi}(H) \rightarrow H^{\chi}, \quad \alpha(h)=\chi^{-1}\left(h_{(1)} \otimes S h_{(5)}\right) \chi\left(h_{(3)} \otimes S h_{(4)}\right) h_{(2)}
$$

is an isomorphism in ${ }_{H}^{{ }^{\chi}} \mathcal{X} \dot{\mathcal{M}}$ with inverse

$$
\alpha^{-1}(h)=\chi^{-1}\left(h_{(1)} S h_{(3)} \otimes h_{(4)}\right) h_{(2)}
$$

and restricts to an isomorphism $\mathcal{F} \chi(\operatorname{ker} \epsilon) \cong \operatorname{ker} \epsilon$.

Proof We deduce this from our theory of twisting of first order differential calculi. Using Proposition 3.2, we know that $(V \otimes H)^{\chi}=\mathcal{G}^{\chi}(V \otimes H)$ is a differential calculus over $H^{\chi}$ and hence of the form $V^{\chi} \otimes H^{\chi}$ for some $V^{\chi}$ a quotient of $\operatorname{ker} \epsilon \subset H^{\chi}$. This $V^{\chi}$ is the right-invariant subspace of the corresponding differential calculus. On the other hand, the projection for the subspace $V$ can be obtained explicitly from its associated calculus by $\pi(a)=\mathrm{d} a_{(1)} \cdot S a_{(2)}$, and similarly for $\pi^{\chi}$ using the Hopf algebra $H^{\chi}$. Thus, from Proposition 3.2, we find,

$$
\begin{aligned}
\pi^{\chi}(a) & =\mathrm{d} a_{(1)} \bullet S^{\chi} a_{(2)} \\
& =\mathrm{d} a_{(1)} \bullet S a_{(3)} U\left(a_{(2)}\right) U^{-1}\left(a_{(4)}\right) \\
& =\chi\left(a_{(1)} \otimes S a_{(7)}\right) \mathrm{d} a_{(2)} \cdot S a_{(6)} \chi^{-1}\left(a_{(3)} \otimes S a_{(5)}\right) U\left(a_{(4)}\right) U^{-1}\left(a_{(8)}\right)
\end{aligned}
$$




$$
\begin{aligned}
& =\chi\left(a_{(1)} \otimes S a_{(4)}\right) \mathrm{d} a_{(2)} \cdot S a_{(3)} U^{-1}\left(a_{(5)}\right) \\
& =\chi^{-1}\left(a_{(1)} S a_{(4)} \otimes a_{(5)}\right) \mathrm{d} a_{(2)} \cdot S a_{(3)} \\
& =\pi\left(\chi^{-1}\left(a_{(1)} S a_{(3)} \otimes a_{(4)}\right) a_{(2)}\right)
\end{aligned}
$$

as stated. In particular, we apply these arguments to the universal differential calculus, which corresponds to $V=\operatorname{ker} \epsilon \subset H$ and hence obtain $\alpha^{-1}$ as a linear isomorphism on ker $\epsilon$. By construction it must in fact identify $\mathcal{F} \chi(\operatorname{ker} \epsilon)$ as a crossed module in ${ }_{H \chi}^{{ }^{\chi}} \dot{\mathcal{M}}$ with ker $\epsilon \subset H^{\chi}$. Finally, it is trivial to check that it extends to the whole of $H=\operatorname{ker} \epsilon \oplus k 1$ with $\alpha(1)=1$ by the same formula.

This tells us also that if $V=\operatorname{ker} \epsilon / M$ by some Ad-stable ideal $M$ then the corresponding ideal for $V^{\chi}$ is $M^{\chi}=\alpha \circ \mathcal{F}^{\chi}(M)$. Note that the isomorphism of crossed modules $\alpha: \mathcal{F}(H) \rightarrow H^{\chi}$ is somewhat nontrivial to obtain by normal Hopf algebraic methods; the intertwining of the adjoint coactions alone is a first step introduced (in a dual form) in [6], which was in fact the starting point behind the present paper.

We turn now to some general applications of our twisting theory, beyond the Planck scale Hopf algebra to be studied in the next section. In fact, many interesting quantum groups are related by twisting and our theory allows the construction of their quantum differential calculi one from the other. We give two important general constructions where the quantum group is a twisting of a tensor product quantum group. But for tensor product Hopf algebras it is easy to obtain calculi given calculi on the pieces. Indeed, if $\left(H_{1}, \Omega_{1}\right)$ and $\left(H_{2}, \Omega_{2}^{*}\right)$ are two Hopf algebras equipped with calculi and associated exterior super-Hopf algebras, it is clear that $\Omega^{\circ} \equiv \Omega_{1} \underline{\otimes} \Omega_{2}$ (the super tensor product) is an exterior super-Hopf algebra over $H:=H_{1} \otimes H_{2}$. d $: \Omega \rightarrow \Omega$. is given by the Leibnitz rule. Restriction to degree 1 yields the corresponding construction for first order bicovariant calculi $\Omega^{1}$, i.e. $\Omega^{1}=\Omega_{1}^{1} \otimes H_{2} \oplus H_{1} \otimes \Omega_{2}^{1}$ given $\Omega_{i}^{1}$, with d defined via the Leibnitz rule.

Proposition 3.6 Given first order calculi $\mathrm{d}: H \rightarrow \Omega^{1}$ on a finite-dimensional Hopf algebra $H$ and $\mathrm{d}: H^{* \mathrm{op}} \rightarrow \Omega_{* \mathrm{op}}^{1}$ on $H^{* \mathrm{op}}$, we obtain a calculus on the quantum double $D(H)$ by

$$
\Omega_{D(H)}^{1}=\Omega^{1} \otimes H^{* \mathrm{op}} \oplus H \otimes \Omega_{* \mathrm{op}}^{1}
$$

and the additional bimodule structure

$$
h \mathrm{~d} \phi=\left\langle S h_{(1)}, \phi_{(1)}\right\rangle\left(\mathrm{d} \phi_{(2)}\right) h_{(2)}\left\langle h_{(3)}, \phi_{(3)}\right\rangle, \quad \phi \mathrm{d} h=\left\langle h_{(1)}, \phi_{(1)}\right\rangle\left(\mathrm{d} h_{(2)}\right) \phi_{(2)}\left\langle S h_{(3)}, \phi_{(3)}\right\rangle
$$

for all $h \in H$ and $\phi \in H^{*}$. Here d restricts to the given one on $H, H^{* \mathrm{op}} \subseteq D(H)$.

Proof It is known that the quantum double $D(H)=H \bowtie H^{* o p}$ is a cotwist of $H \otimes H^{* o p}$ by the cocycle

$$
\chi((h \otimes \phi) \otimes(g \otimes \psi))=\epsilon(h)\langle\phi, S g\rangle \epsilon(\psi) .
$$

See [5]. We then apply Proposition 3.2, i.e. the functor $\mathcal{G}^{\chi}$. The vector space, coactions and $\mathrm{d}$ are not changed from the tensor product calculus under the functor, but the bimodule structures are, as shown. These are easily computed from the form of the cocycle.

This is a different and rather more geometrical approach to the construction of the differential calculi on $D(H)$ than the one in [- 1 based on its representation theory. In a similar spirit we may consider the general double cross product Hopf algebra $H \bowtie_{\mathcal{R}} H$ associated to any dualquasitriangular Hopf algebra $H$ [5]. 
Proposition 3.7 Let $H$ be dual-quasitriangular. Given two calculi $\mathrm{d}: H \rightarrow \Omega_{L}^{1}$ and $\mathrm{d}: H \rightarrow$ $\Omega_{R}^{1}$ we obtain a calculus on $H \bowtie_{\mathcal{R}} H$ by

$$
\Omega^{1}=\Omega_{L}^{1} \otimes H \oplus H \otimes \Omega_{R}^{1}
$$

and the additional bimodule structure

$$
h \mathrm{~d} a=\mathcal{R}^{-1}\left(h_{(1)}, a_{(1)}\right)\left(\mathrm{d} a_{(2)}\right) h_{(2)} \mathcal{R}\left(h_{(3)}, a_{(3)}\right), \quad a \mathrm{~d} h=\mathcal{R}\left(h_{(1)}, a_{(1)}\right)\left(\mathrm{d} h_{(2)}\right) a_{(2)} \mathcal{R}^{-1}\left(h_{(3)}, a_{(3)}\right)
$$

for all $h$ in the first copy of $H$ and $a$ in the second. Here $\mathrm{d}$ restricted to each copy of $H$ is the given one.

Proof We proceed in the same fashion as above. The cocycle is given by

$$
\chi((h \otimes a) \otimes(g \otimes b))=\epsilon(h) \mathcal{R}^{-1}(a \otimes g) \epsilon(b), \quad \forall h, g, a, b \in H
$$

and allows us to write $H \bowtie_{\mathcal{R}} H=(H \otimes H)^{\chi}$. See [5]. Here the two copies of $H$ remain sub-Hopf algebras as for the quantum double case above, and the remaining computation is similar.

In particular, a standard formulation of the q-Lorentz group is as $S U_{q}(2) \bowtie_{\mathcal{R}} S U_{q}(2)$, a twist of the q-Euclidean rotation group as $S U_{q}(2) \otimes S U_{q}(2)$. Usually the calculi on these are obtained and studied separately, but the above proposition constructs one from the other. This extends the 'quantum Wick rotation' in [17] to the construction of bicovariant differential calculi. In both the above constructions the factors appear as sub(Hopf) algebras and in this case there is a well-defined notion of a calculus being decomposable or built up from calculi on the factors; the above results fully classify such decomposable calculi.

\section{Planck scale Hopf algebra as a cotwist and its differential geometry}

The remainder of the paper applies the preceding results to one particular Hopf algebra, namely the bicrossproduct Hopf algebra $\mathbb{C}[x] \triangleleft_{\hbar, \mathrm{G}} \mathbb{C}[p]$ introduced in [7]. For the purposes of the present section we work algebraically with $g=e^{-\frac{x}{G}}$ and $g^{-1}$ instead of $x$. Then the explicit formulae (as stated in the introduction) are

$$
\begin{gathered}
{[p, g]=\imath A(1-g) g,} \\
\Delta p=p \otimes g+1 \otimes p, \quad \Delta g=g \otimes g, \\
S p=-p g^{-1}, \quad \epsilon p=0, \quad S g=g^{-1}, \quad \epsilon g=1,
\end{gathered}
$$

where $A=\frac{\hbar}{\mathrm{G}}$. Also, as $\hbar \rightarrow 0$ (corresponding to $A \rightarrow 0$ ), we obtain $C\left(B_{+}\right)$(in an algebraic form) where

$$
B_{+}=\mathbb{R} \propto_{\mathrm{G}} \mathbb{R}, \quad(x, p)\left(x^{\prime}, p^{\prime}\right)=\left(x+x^{\prime}, p e^{-\frac{x^{\prime}}{\mathrm{G}}}+p^{\prime}\right)
$$

as explained in [7]. We consider $B_{+}$to be the classical phase space underlying the quantum system described by the Plank-scale Hopf algebra. In terms of the exponentiated coordinate in place of $x$, the group law of $B_{+}$is that of a matrix group

$$
\left(\begin{array}{ll}
g & 0 \\
p & 1
\end{array}\right)\left(\begin{array}{ll}
g^{\prime} & 0 \\
p^{\prime} & 1
\end{array}\right)=\left(\begin{array}{cc}
g g^{\prime} & 0 \\
p g^{\prime}+p^{\prime} & 1
\end{array}\right)
$$

The notation $\mathbb{C}[x] \bowtie_{\hbar, \mathrm{G}} \mathbb{C}[p]$ reflects the construction of this Hopf algebra as a bicrossproduct, i.e. a semidirect product as an algebra and a semidirect coproduct as a coalgebra by certain actions and coactions arising from a Lie group factorisation [18] [19] [7]. This aspect will be used extensively in Section 5 . 


\subsection{The cocycle and differential calculi}

Our starting points are the known facts that the Hopf algebra $\mathbb{C}[x] \bowtie_{\hbar, \mathrm{G}} \mathbb{C}[p]$ is of a self-dual form and at the same time a twisting (of the coproduct) of $U\left(\mathfrak{b}_{+}\right)$where $\mathfrak{b}_{+}$is the Borel subalgebra of $s l_{2}$. Combining these observations, one may expect that it is also a product twist by a cocycle. This turns out to be the case.

Proposition $4.1 \chi$ defined by

$$
\chi=(\epsilon \otimes \epsilon) \circ \exp \left(\imath A \frac{\partial}{\partial p} \otimes \frac{\partial}{\partial g^{-1}}\right), \quad \chi^{-1}=(\epsilon \otimes \epsilon) \circ \exp \left(\imath A \frac{\partial}{\partial p} \otimes \frac{\partial}{\partial g}\right)
$$

is a unital 2-cocycle on $C\left(B_{+}\right)$, and $\mathbb{C}[x] \bowtie_{\hbar, \mathrm{G}} \mathbb{C}[p]=C\left(B_{+}\right)^{\chi}$.

Proof In order to show that $\chi$ is a unital 2-cocycle we have to show its invertibility, the cocycle condition and the unitality (see Preliminaries). It will be useful to have the explicit expressions of $\chi$ and $\chi^{-1}$ on a basis $\left\{p^{n} g^{r} \mid n \in \mathbb{N}_{0}, r \in \mathbb{Z}\right\}$ of $C\left(B_{+}\right)$:

$$
\chi\left(p^{n} g^{r} \otimes p^{m} g^{s}\right)=\delta_{m, 0}(\imath A)^{n} \prod_{k=0}^{n-1}(-s-k), \quad \chi^{-1}\left(p^{n} g^{r} \otimes p^{m} g^{s}\right)=\delta_{m, 0}(\imath A)^{n} \prod_{k=0}^{n-1}(s-k) .
$$

For the invertibility we require

$$
\chi\left(a_{(1)} \otimes b_{(1)}\right) \chi^{-1}\left(a_{(2)} \otimes b_{(2)}\right)=\epsilon(a) \epsilon(b), \quad \chi^{-1}\left(a_{(1)} \otimes b_{(1)}\right) \chi\left(a_{(2)} \otimes b_{(2)}\right)=\epsilon(a) \epsilon(b) .
$$

To see this we take $a=p^{n} g^{r}$ and $b=p^{m} g^{s}$ and the first expression becomes

$$
\begin{aligned}
& \sum_{k, l}\left(\begin{array}{l}
n \\
k
\end{array}\right)\left(\begin{array}{c}
m \\
l
\end{array}\right) \chi\left(p^{k} g^{r} \otimes p^{l} g^{s}\right) \chi^{-1}\left(p^{n-k} g^{k+r} \otimes p^{m-l} g^{l+s}\right) \\
& =\sum_{k, l}\left(\begin{array}{l}
n \\
k
\end{array}\right)\left(\begin{array}{c}
m \\
l
\end{array}\right) \delta_{l, 0} \delta_{m, l}(\imath A)^{n} \prod_{i=0}^{k-1}(-s-i) \prod_{j=0}^{n-k}(l+s-j) \\
& =\delta_{m, 0}(\imath A)^{n} \sum_{k}\left(\begin{array}{l}
n \\
k
\end{array}\right) \prod_{i=0}^{k-1}(-s-i) \prod_{j=0}^{n-k}(s-j) \\
& =\delta_{m, 0} \delta_{n, 0}=\epsilon\left(p^{n} g^{r}\right) \epsilon\left(p^{m} g^{s}\right) .
\end{aligned}
$$

We have used

$$
\sum_{k=0}^{n}\left(\begin{array}{l}
n \\
k
\end{array}\right) \prod_{i=0}^{k-1}(-s-i) \prod_{j=0}^{n-k}(s-j)=\delta_{n, 0}, \quad \forall s \in \mathbb{Z} \forall n \in \mathbb{N}_{0}
$$

While this is obvious for $s=0$ it follows easily by induction for $s \neq 0$. Note that the exchange of $\chi$ and $\chi^{-1}$ in the above calculation is equivalent to replacing $s$ by $-s$. Thus follows the second equation as well. Next, for the cocycle condition we take $p^{n} g^{r}, p^{m} g^{s}, p^{l} g^{t}$ for $h, g, f$ in (3). The 
left hand side evaluates to

$$
\begin{aligned}
& \sum_{j, k}\left(\begin{array}{c}
m \\
j
\end{array}\right)\left(\begin{array}{l}
l \\
k
\end{array}\right) \chi\left(p^{j} g^{s} \otimes p^{k} g^{t}\right) \chi\left(p^{n} g^{r} \otimes p^{m-j+l-k} g^{j+s+k+t}\right) \\
& =\sum_{j, k}\left(\begin{array}{c}
m \\
j
\end{array}\right)\left(\begin{array}{l}
l \\
k
\end{array}\right) \delta_{k, 0} \delta_{m-j+l-k, 0}(\imath A)^{j+n} \prod_{i=0}^{j-1}(-t-i) \prod_{h=0}^{n-1}(-j-s-k-t-h) \\
& =\sum_{j}\left(\begin{array}{c}
m \\
j
\end{array}\right) \delta_{m-j+l, 0}(\imath A)^{j+n} \prod_{i=0}^{j-1}(-t-i) \prod_{h=0}^{n-1}(-j-s-t-h) \\
& =\delta_{l, 0}(\imath A)^{n+m} \prod_{i=0}^{m-1}(-t-i) \prod_{h=0}^{n-1}(-m-s-t-h) .
\end{aligned}
$$

The right hand side is

$$
\begin{aligned}
& \sum_{j, k}\left(\begin{array}{l}
n \\
j
\end{array}\right)\left(\begin{array}{l}
m \\
k
\end{array}\right) \chi\left(p^{j} g^{r} \otimes p^{k} g^{s}\right) \chi\left(p^{n-j+m-k} g^{j+r+k+s} \otimes p^{l} g^{t}\right) \\
& =\sum_{j, k}\left(\begin{array}{c}
n \\
j
\end{array}\right)\left(\begin{array}{l}
m \\
k
\end{array}\right) \delta_{k, 0} \delta_{l, 0}(\imath A)^{n+m-k} \prod_{i=0}^{j-1}(-s-i) \prod_{h=0}^{n-j+m-k-1}(-t-h) \\
& =\delta_{l, 0}(\imath A)^{n+m} \sum_{j}\left(\begin{array}{c}
n \\
j
\end{array}\right) \prod_{i=0}^{j-1}(-s-i) \prod_{h=0}^{n-j+m-1}(-t-h) \\
& =\delta_{l, 0}(\imath A)^{n+m} \prod_{i=0}^{m-1}(-t-i) \prod_{h=0}^{n-1}(-m-s-t-h) .
\end{aligned}
$$

The last equality can be easily checked by induction in $n$. Finally, the unitality in (3) follows easily from the explicit formula for $\chi$. It remains to check that the twist of $C\left(B_{+}\right)$defined by $\chi$ is indeed $\mathbb{C}[x] \triangleleft_{\hbar, \mathrm{G}} \mathbb{C}[p]$. For that, it is sufficient to check the commutator between $p$ and $g$. For clarity, we distinguish the twisted product from the untwisted one by denoting the former with a •.

$$
\begin{aligned}
g \bullet g & =\chi(g \otimes g) g g \chi^{-1}(g \otimes g)=g g \\
p \bullet g & =\chi(p \otimes g) g g \chi^{-1}(g \otimes g)+\chi(1 \otimes g) p g \chi^{-1}(g \otimes g)+\chi(1 \otimes g) g \chi^{-1}(p \otimes g) \\
& =-\imath A g g+p g+\imath A g \\
g \bullet p & =\chi(g \otimes p) g g \chi^{-1}(g \otimes g)+\chi(g \otimes 1) g p \chi^{-1}(g \otimes g)+\chi(g \otimes 1) g \chi^{-1}(g \otimes p)=g p
\end{aligned}
$$

In particular we obtain

$$
p \bullet g-g \bullet p=\imath A(1-g) g=\imath A(1-g) \bullet g,
$$

which is the correct relation in $\mathbb{C}[x] \triangleleft_{\hbar, \mathrm{G}} \mathbb{C}[p]$.

Let us now turn to the differential calculi on $\mathbb{C}[x] \triangleleft_{\hbar, \mathrm{G}} \mathbb{C}[p]$. We first repeat a result from [10] about the untwisted Hopf algebra $C\left(B_{+}\right)$.

Proposition 4.2 10 (a) Finite dimensional differential calculi $\Omega^{1}$ on $C\left(B_{+}\right)$are in one-to-one correspondence to non-empty finite sets $I \subset \mathbb{N}$ and have dimension $\left(\sum_{n \in I} n\right)-1$. 
(b) The differential calculus of dimension $n \geq 2$ corresponding to $\{n, 1\}$ has a right invariant basis $\eta_{0}, \ldots \eta_{n-1}$ so that

$$
\begin{gathered}
\mathrm{d} g=g \eta_{0}, \quad \mathrm{~d} g=g \eta_{1}, \\
{\left[g, \eta_{k}\right]=0, \quad\left[p, \eta_{k}\right]= \begin{cases}0 & \text { if } k=0 \text { or } k=n-1 \\
g \eta_{k+1} & \text { if } 0<k<n-1\end{cases} } \\
\beta_{L}\left(\eta_{k}\right)= \begin{cases}g^{-k} \otimes \eta_{k} & \text { if } k \neq 1 \\
g^{-1} \otimes \eta_{1}+g^{-1} p \otimes \eta_{0} & \text { if } k=1\end{cases}
\end{gathered}
$$

(c) The differential calculus of dimension $n-1 \geq 1$ corresponding to $\{n\}$ is the same as (b) except that $\eta_{0}=0$.

Proof We refer to [10, Prop. 3.5]. Note however, that in [10] different generators were used for $C\left(B_{+}\right)$, so that the coproduct appeared in a different form. In our conventions the crossed submodule $M \subset$ ker $\epsilon$ corresponding to $\{n, 1\}$ is generated by $(g-1)(g-1), p(g-1), \ldots, p^{n}$ as a crossed module. Denoting the equivalence classes of $g-1, p, \ldots, p^{n-1}$ in $\operatorname{ker} \epsilon / M$ by $\eta_{0}, \ldots, \eta_{n-1}$, we obtain the derivative and commutation relations as stated. For the left coaction note that the left adjoint action on $C\left(B_{+}\right)$takes the form

$$
\operatorname{Ad}_{\mathrm{L}}\left(f(g) p^{k}\right)=\sum_{t=0}^{k}\left(\begin{array}{l}
k \\
t
\end{array}\right) g^{-k} p^{k-t} \otimes f(g)(g-1)^{k-t} p^{t} .
$$

$\beta$ is then obtained by composition with the projection to $\operatorname{ker} \epsilon / M$.

We can now apply our twisting theory in Sections 2,3 to solve the classification problem for calculi on the Planck scale Hopf algebra,

Proposition 4.3 (a) Finite dimensional differential calculi $\Omega^{1}$ on $\mathbb{C}[x] \triangleleft_{\hbar, G} \mathbb{C}[p]$ are in one-toone correspondence to non-empty finite subsets $I \subset \mathbb{N}$ with dimensions as in Proposition 4.9 .

(b) The differential calculus of dimension $n \geq 2$ corresponding to $\{n, 1\}$ has a right invariant basis $\eta_{0}, \ldots \eta_{n-1}$ so that

$$
\begin{gathered}
\mathrm{d} g=g \eta_{0}, \quad \mathrm{~d} p=g \eta_{1}, \\
{\left[g, \eta_{k}\right]=\left\{\begin{array}{ll}
0 & \text { if } k \neq 1 \\
\imath A g \eta_{0} & \text { if } k=1
\end{array} \quad\left[p, \eta_{k}\right]=\imath A k g \eta_{k}+ \begin{cases}0 & \text { if } k=0 \text { or } k=n-1 \\
g \eta_{k+1} & \text { if } 0<k<n-1\end{cases} \right.}
\end{gathered}
$$

and $\beta$ of the same form as in Proposition 4.2 .

(c) The differential calculus of dimension $n-1 \geq 1$ corresponding to $\{n\}$ is the same as (b) except that $\eta_{0}=0$.

Proof We apply Proposition 3.2 to Proposition 4.2. Part (a) remains unchanged. For part (b) we calculate the twisted actions in terms of the untwisted ones (using a $\bullet$ to denote the twisted ones).

$$
\begin{aligned}
& g \bullet \eta_{k}=\chi\left(g \otimes g^{-k}\right) g \eta_{k}+\delta_{k, 1} \chi\left(g \otimes g^{-1} p\right) g \eta_{0}=g \eta_{k} \\
& \eta_{k} \bullet g=\chi\left(g^{-k} \otimes g\right) \eta_{k} g+\delta_{k, 1} \chi\left(g^{-1} p \otimes g\right) \eta_{0} g=\eta_{k} g-\imath A \delta_{k, 1} \eta_{0} g \\
& p \bullet \eta_{k}=\chi\left(p \otimes g^{-k}\right) g \eta_{k}+\chi\left(1 \otimes g^{-k}\right) p \eta_{k}+\delta_{k, 1}\left(\chi\left(p \otimes g^{-1} p\right) g \eta_{0}+\chi\left(1 \otimes g^{-1} p\right) p \eta_{0}\right)
\end{aligned}
$$




$$
\begin{aligned}
& =\imath A k g \eta_{k}+p \eta_{k} \\
\eta_{k} \bullet p & =\chi\left(g^{-k} \otimes p\right) \eta_{k} g+\chi\left(g^{-k} \otimes 1\right) \eta_{k} p+\delta_{k, 1}\left(\chi\left(g^{-1} p \otimes p\right) \eta_{0} g+\chi\left(g^{-1} p \otimes 1\right) \eta_{0} p\right)=\eta_{k} p
\end{aligned}
$$

This gives the new commutators and the expressions for the differentials. For the coaction we observe that $g^{-1} \bullet p=g^{-1} p$ so that its form does not change. Part (c) remains unchanged.

For the remainder of the section we concentrate on the calculus $\{2,1\}$ which is the quantisation of the standard classical calculus on $B_{+}$. We can use the twisting theory to quantise in fact the entire exterior algebra in this case.

Proposition 4.4 The exterior algebra $\Omega$ of $\mathbb{C}[x] \bowtie_{\hbar, \mathrm{G}} \mathbb{C}[p]$ corresponding via twisting to the classical one of $C\left(B_{+}\right)$has the following properties. The first order calculus has a basis $\{\xi, \eta\}$ of right-invariant 1-forms with

$$
\begin{gathered}
\mathrm{d} g=g \xi, \quad \mathrm{d} p=g \eta, \quad[a, \xi]=0, \quad[a, \eta]=\imath A \mathrm{~d} a, \quad \forall a \in \mathbb{C}[x] \triangleleft_{\hbar, \mathrm{G}} \mathbb{C}[p], \\
\beta_{L}(\xi)=1 \otimes \xi, \quad \beta_{L}(\eta)=g^{-1} \otimes \eta+g^{-1} p \otimes \xi .
\end{gathered}
$$

The 2-forms have relations

$$
\begin{gathered}
\xi \wedge \xi=0, \quad \eta \wedge \xi=-\xi \wedge \eta, \quad \eta \wedge \eta=\imath A \xi \wedge \eta, \\
\mathrm{d} \xi=0, \quad \mathrm{~d} \eta=\eta \wedge \xi .
\end{gathered}
$$

As a $\mathbb{Z}_{2}$-graded Hopf algebra $\Omega$ has the structure

$$
\begin{gathered}
\Delta \xi=\xi \otimes 1+1 \otimes \xi, \quad \Delta \eta=g^{-1} \otimes \eta+g^{-1} p \otimes \xi-\eta \otimes 1, \\
\epsilon(\xi)=\epsilon(\eta)=0, \quad S \xi=-\xi, \quad S \eta=-g \eta+p \xi .
\end{gathered}
$$

Proof For the first order calculus we define $\xi \equiv \eta_{0}$ and $\eta \equiv \eta_{1}$. The commutation relations in the $n=2$ case (b) of Proposition 4.3 become as stated. Next, the classical space of 2 -forms on $C\left(B_{+}\right)$is spanned by $\xi \wedge \eta=-\eta \wedge \xi$. Denoting the wedge product on $\mathbb{C}[x] \bowtie_{\hbar, \mathrm{G}} \mathbb{C}[p]$ by $\wedge$. we have

$$
\begin{aligned}
\eta \wedge \bullet \eta= & \chi\left(g^{-1} \otimes g^{-1}\right) \eta \wedge \eta+\chi\left(g^{-1} p \otimes g^{-1}\right) \xi \wedge \eta \\
& +\chi\left(g^{-1} \otimes g^{-1} p\right) \eta \wedge \xi+\chi\left(g^{-1} p \otimes g^{-1} p\right) \xi \wedge \xi \\
= & \imath A \xi \wedge \eta
\end{aligned}
$$

The other wedge products involving $\xi$ and $\eta$ are identical to the classical ones due to the biinvariance of $\xi$. This leads to the relations stated. Finally, for the differentials of the 1-forms observe (the twisted and untwisted wedge products are the same here)

$$
\begin{aligned}
\mathrm{d} \xi & =\mathrm{d}\left(g^{-1} \mathrm{~d} g\right)=\mathrm{d} g^{-1} \wedge \mathrm{d} g=-g^{-1} \xi \wedge g \xi=0, \\
\mathrm{~d} \eta & =\mathrm{d}\left(g^{-1} \mathrm{~d} p\right)=\mathrm{d} g^{-1} \wedge \mathrm{d} p=-g^{-1} \xi \wedge g \eta=\eta \wedge \xi .
\end{aligned}
$$

The coproduct and antipode are readily obtained using Proposition 2.1. The exterior algebra here coincides with the Woronowicz prolongation of the first order part.

In terms of generators $x$ and $p$ the exterior algebra is generated by $\mathrm{d} x, \mathrm{~d} p$ with the relations

$$
a \mathrm{~d} x=(\mathrm{d} x) a, \quad a \mathrm{~d} p=(\mathrm{d} p) a+\frac{\imath \hbar}{\mathrm{G}} \mathrm{d} a,
$$




$$
\mathrm{d} x \wedge \mathrm{d} x=0, \quad \mathrm{~d} x \wedge \mathrm{d} p=-\mathrm{d} p \wedge \mathrm{d} x, \quad \mathrm{~d} p \wedge \mathrm{d} p=0 .
$$

From this we see explicitly that in the classical limit $\hbar \rightarrow 0$ we obtain the usual exterior algebra on $B_{+}$. By contrast, the other limit $\mathrm{G} \rightarrow 0$ is highly singular with these generators, so that the exterior algebra is not even defined in this case. In other words, the presence of 'gravity' in the form of $\mathrm{G}$ restores the geometrical picture not visible in flat space quantum mechanics.

We also know from the Preliminaries that associated to a first order calculus is a quantum tangent space. The right-invariant derivatives are generated by elements of $L$ dual to $V$ and obey a braided Leibniz rule.

Proposition 4.5 Let $\left\{\xi^{*}, \eta^{*}\right\}$ be the basis of $L$ dual to the basis $\{\xi, \eta\}$ above. Then,

$$
\begin{aligned}
& \partial_{\xi}(: f(g, p):)=: g \frac{\partial}{\partial g} f(g, p+\imath A)+g(f(g, p+\imath A)-f(g, p)):, \\
& \partial_{\eta}(: f(g, p):)=: g \frac{f(g, p+\imath A)-f(g, p)}{\imath A}:
\end{aligned}
$$

Proof We observe that $\mathrm{d} g^{n}=(\mathrm{d} g) n g^{n-1}$ and $\mathrm{d} p^{n}=(\mathrm{d} p) \frac{(p+\imath A)^{n}-p^{n}}{\imath A}$ (this can be easily checked by induction), so that

$$
\begin{aligned}
\mathrm{d}\left(g^{n} p^{m}\right) & =\left(\mathrm{d} g^{n}\right) p^{m}+g^{n} \mathrm{~d} p^{m} \\
& =\left(\mathrm{d} g^{n}\right) p^{m}+g^{n}(\mathrm{~d} p) \frac{(p+\imath A)^{m}-p^{m}}{\imath A} \\
& =\left(\mathrm{d} g^{n}\right) p^{m}+(\mathrm{d} p) g^{n} \frac{(p+\imath A)^{m}-p^{m}}{\imath A}+\imath A\left(\mathrm{~d} g^{n}\right) \frac{(p+\imath A)^{m}-p^{m}}{\imath A} \\
& =\left(\mathrm{d} g^{n}\right)(p+\imath A)^{m}+(\mathrm{d} p) g^{n} \frac{(p+\imath A)^{m}-p^{m}}{\imath A} \\
& =\xi n g^{n}(p+\imath A)^{m}+(\eta g+\imath A \xi g) g^{n} \frac{(p+\imath A)^{m}-p^{m}}{\imath A} \\
& =\xi\left(n g^{n}(p+\imath A)^{m}+g^{n+1}\left((p+\imath A)^{m}-p^{m}\right)\right)+\eta g^{n+1} \frac{(p+\imath A)^{m}-p^{m}}{\imath A},
\end{aligned}
$$

which we compare with the property $\mathrm{d} f=\xi \partial_{\xi}(f)+\eta \partial_{\eta}(f)$ of the partial derivatives.

In terms of coordinates $x, p$ we can similarly write the action of the basis of $L$ dual to $\{\mathrm{d} x, \eta\}$ as

$$
\begin{aligned}
& \partial_{x}(: f(x, p):)=: \frac{\partial}{\partial x} f\left(x, p+\frac{\imath \hbar}{\mathrm{G}}\right)-\frac{e^{-\frac{x}{\mathrm{G}}}}{\mathrm{G}}\left(f\left(x, p+\frac{\imath \hbar}{\mathrm{G}}\right)-f(x, p)\right):, \\
& \partial_{\eta}(: f(x, p):)=\frac{\mathrm{G}}{\imath \hbar}: e^{-\frac{x}{\mathrm{G}}}\left(f\left(x, p+\frac{\imath \hbar}{\mathrm{G}}\right)-f(x, p)\right):
\end{aligned}
$$

(here $\partial_{x}$ denotes the action of the basis element dual to $\mathrm{d} x$ by a slight abuse of notation).

Finally, for completeness we note that all these formulae are for right-invariant differential forms. There is an equally good theory based on $L, V \in \dot{\mathcal{M}}_{H}^{H}$ and left-invariant partial derivatives. We take a left-invariant basis of the 1 -forms to be $\left\{\xi=g^{-1} \mathrm{~d} g, \bar{\eta}=\mathrm{d} p-p g^{-1} \mathrm{~d} g\right\}$. The relations of the the calculus become

$$
\begin{gathered}
{[a, \xi]=0, \quad[a, \bar{\eta}]=\imath A \mathrm{~d} a, \quad \forall a \in \mathbb{C}[x] 凶_{\hbar, \mathrm{G}} \mathbb{C}[p],} \\
\beta_{R}(\xi)=\xi \otimes 1, \quad \beta_{R}(\bar{\eta})=\bar{\eta} \otimes g-\xi \otimes p,
\end{gathered}
$$




$$
\xi \wedge \xi=0, \quad \bar{\eta} \wedge \xi=-\xi \wedge \bar{\eta}, \quad \bar{\eta} \wedge \bar{\eta}=\imath A \bar{\eta} \wedge \xi
$$

Moreover, the differential in $\Omega$ is generated by (graded) commutation with the element $\theta=$ $-\frac{1}{2}(\eta+\bar{\eta})$ as

$$
[\theta, \alpha]=\imath A \mathrm{~d} \alpha, \quad \forall \alpha \in \Omega .
$$

This is a step towards a Connes spectral triple description of this calculus, to be considered elsewhere. The generator $\frac{1}{\imath A} \theta$ is singular in the limit $A \rightarrow 0(\hbar \rightarrow 0)$ so that the presence of $\hbar$ allows for nicer properties of the differential calculus than visible classically. This is a typical feature of q-deformation known for calculi on standard quantum groups.

We likewise bar the braided derivations in the left-invariant version of the theory to avoid confusion with the above right-invariant ones. The left-invariant derivations corresponding to $\{\mathrm{d} x, \bar{\eta}\}$ in the sense $\mathrm{d} f=\left(\bar{\partial}_{x} f\right) \mathrm{d} x+\left(\bar{\partial}_{\eta} f\right) \bar{\eta}$ are

$$
\begin{gathered}
\bar{\partial}_{x}(: f(x, p):)=: \frac{\partial}{\partial x} f(x, p)+\frac{p}{\imath \hbar}\left(f\left(x, p-\frac{\imath \hbar}{\mathrm{G}}\right)-f(x, p)\right): \\
\bar{\partial}_{\eta}(: f(x, p):)=-\frac{\mathrm{G}}{\hbar}:\left(f\left(x, p-\frac{\hbar}{\mathrm{G}}\right)-f(x, p)\right):
\end{gathered}
$$

\subsection{Quantum Poisson bracket}

We conclude this section with some elements of 'quantum Poisson geometry'. We recall first of all the classical situation. In fact, for any twisting of a commutative Hopf algebra $H$ by a cocycle $\chi$ admitting a reasonable expansion in a parameter $\hbar$ (so that the deformation is flat) one knows on general grounds that the commutative Hopf algebra is (an algebraic version of) a Poisson-Lie group. As for any flat deformation, the Poisson bracket is provided by the $\hbar \rightarrow 0$ part of $\frac{\imath}{\hbar}[$,$] (the leading part of the commutator). When H=\mathbb{C}[G]$ is an algebraic group of Lie type one can say rather more. We let $\mathfrak{g}$ be the Lie algebra of $G$. If the cocycle $\chi_{\hbar}$ has the form

$$
\chi_{\hbar}(a \otimes b)=a(e) b(e)+\frac{\hbar}{\imath}\langle\tilde{\sigma}, \mathrm{d} a \otimes \mathrm{d} b\rangle(e)+\mathcal{O}\left(\hbar^{2}\right)
$$

where $\sigma \in \mathfrak{g} \otimes \mathfrak{g}$ and $\tilde{\sigma}$ denotes the extension as a left-invariant bivector field. Then it is known from Drinfeld's theory [3] that

$$
r=\sigma-\tau(\sigma)
$$

( $\tau$ the flip map) is a triangular solution of the Classical Yang-Baxter equation. Moreover,

$$
\{a, b\}=\left\langle\tilde{r}-\tilde{r}^{R}, \mathrm{~d} a \otimes \mathrm{d} b\right\rangle
$$

is the Poisson bracket of which $H^{\chi}$ is the quantisation, and which makes $G$ a Poisson-Lie group. [3] proves in fact the converse to this (the formal existence of $\chi_{\hbar}$ ) but the above is also covered. In our case of the Planck scale Hopf algebra, $G=B_{+}$with Lie algebra $\mathfrak{b}_{+}$spanned by $x_{0}, x_{1}$ with relations $\left[x_{0}, x_{1}\right]=\frac{\imath}{\mathrm{G}} x_{1}$ becomes a triangular Lie bialgebra with

$$
r=x_{0} \otimes x_{1}-x_{1} \otimes x_{0} .
$$

The resulting Lie bialgebra has the Lie bicross sum form $₫$ of which the bicrossproduct Hopf algebras are quantisations, see 20]. Note that the corresponding Poisson bracket, as with all (quasi)triangular Poisson-Lie groups, cannot be symplectic since it must vanish at least at the group identity. 
Proposition 4.6 The Poisson bracket on $C\left(B_{+}\right)$, for which the cocycle $\chi$ of Proposition 4.1 provides the quantisation, is

$$
\{a, b\}=\left(e^{-\frac{x}{G}}-1\right)\left(\frac{\partial a}{\partial x} \frac{\partial b}{\partial p}-\frac{\partial b}{\partial x} \frac{\partial a}{\partial p}\right) .
$$

Proof Expanding $\chi$ of Proposition 4.1 in $\hbar$ and expressing everything in terms of the coordinates $x, p$ yields

$$
\begin{aligned}
a \bullet b= & a b+\imath \hbar \epsilon\left(\frac{\partial}{\partial p} a_{(1)}\right) \epsilon\left(e^{-\frac{x}{G}} \frac{\partial}{\partial x} b_{(1)}\right) a_{(2)} b_{(2)} \\
& -\imath \hbar a_{(1)} b_{(1)} \epsilon\left(\frac{\partial}{\partial p} a_{(2)}\right) \epsilon\left(e^{\frac{x}{G}} \frac{\partial}{\partial x} b_{(2)}\right)+\mathcal{O}\left(\hbar^{2}\right) \\
= & a b+\imath \hbar\left(e^{-\frac{x}{G}} \frac{\partial a}{\partial p} \frac{\partial b}{\partial x}-\frac{\partial a}{\partial p} \frac{\partial b}{\partial x}\right)+\mathcal{O}\left(\hbar^{2}\right) \\
= & a b+\imath \hbar\left(e^{-\frac{x}{G}}-1\right)\left(\frac{\partial a}{\partial p} \frac{\partial b}{\partial x}\right)+\mathcal{O}\left(\hbar^{2}\right) \\
a \bullet b-b \bullet a= & \frac{\hbar}{\imath}\left(e^{-\frac{x}{G}}-1\right)\left(\frac{\partial a}{\partial x} \frac{\partial b}{\partial p}-\frac{\partial b}{\partial x} \frac{\partial a}{\partial p}\right)+\mathcal{O}\left(\hbar^{2}\right) .
\end{aligned}
$$

For the general quantum group formulation, we work over a general field $k$. Since $L, V \in{ }_{H}^{H} \dot{\mathcal{M}}$ we can take their arbitrary tensor powers to define tensor fields of arbitrary mixed rank using the same correspondence with bicovariant bimodules. Thus $\Omega^{-1}=L \otimes H$ and $\Omega^{-1} \otimes_{H} \Omega^{-1}=$ $L \otimes L \otimes H$ etc. We have a super-Hopf algebra $T_{-1}\left(\Omega^{-1}\right)$ and a theory of twisting of of quantum vector fields using the same theory of Section 2. Also, since morphisms in ${ }_{H}^{H} \dot{\mathcal{M}}$ induce morphisms between bicovariant bimodules, the evaluation map $\langle\rangle:, L \otimes V \rightarrow k$ induces the pairing between vector fields and 1 -forms. Thus

$$
\begin{gathered}
\Omega^{-1} \underset{H}{\otimes} \Omega^{1} \rightarrow H, \quad\langle x \otimes h, v \otimes g\rangle=\left\langle x, h_{(1)} \triangleright v\right\rangle h_{(2)} g, \\
\Omega^{-1} \stackrel{H}{\otimes}_{\Omega^{-1}}^{\otimes} \Omega^{1} \rightarrow \Omega^{-1}, \quad\langle x \otimes y \otimes h, v \otimes g\rangle=x \otimes\left\langle y, h_{(1)} \triangleright v\right\rangle h_{(2)} g, \\
\Omega^{-1}{\underset{H}{\otimes}}_{\Omega_{H}^{-1}}^{\otimes} \Omega_{H}^{1} \underset{H}{\otimes} \Omega^{1}, \quad\langle x \otimes y \otimes h, v \otimes w \otimes g\rangle=\left\langle y, h_{(1)} \triangleright v\right\rangle\left\langle x, h_{(2)} \triangleright w\right\rangle h_{(3)} g,
\end{gathered}
$$

etc. The pairing $L \otimes L \otimes V \otimes V \rightarrow k$ in (19) is the natural one in a braided category, namely to evaluate the inner $L \otimes V$ first and then the outer. The resulting pairing is also the same as applying (18) to the first factor of $\Omega^{1} \otimes_{H} \Omega^{1}$ to obtain an element of $\Omega^{-1} \otimes_{H} \Omega^{1}$ and then applying (17).

This is not the only way to formulate vector fields (for example a more left-right symmetric way is to consider $L \in \dot{\mathcal{M}}_{H}^{H}$ and $\Omega^{-1}=H \otimes L$, extending the pairing by $\left.\langle h \otimes x, v \otimes g\rangle=h\langle x, v\rangle g\right)$ but it is the one natural in the context of the Woronowicz exterior algebra (which can be viewed as based on a fixed identification of bicovariant bimodules with ${ }_{H}^{H} \dot{\mathcal{M}}$ (say)). Taking now $\Omega^{n}$ defined by quotients of $V^{\otimes n}$ in the exterior algebra in this approach, the natural definition of antisymmetric vector fields is as corresponding to the appropriate subspace of $L^{\otimes n}$ dual to this quotient. In particular, the Poisson bivector field should be an element

$$
\Pi \in \Omega^{-2}=\left\{x \otimes y-\Psi_{L, L}(x \otimes y) \mid x, y \in L\right\} \otimes H
$$


since $V \otimes V$ is quotiented by $\operatorname{ker}\left(\mathrm{id}-\Psi_{V, V}\right)$ in degree 2 .

In general, we also need to impose a 'Jacobi identity' on $\Pi$, which can be done as follows at least in the nice case where the quantum Poisson bracket is non-degenerate: we can consider $\Pi$ by the above as a map $\Omega^{1} \rightarrow \Omega^{-1}$ and demand that it is invertible, and that the inverse corresponds to evaluation against some $\omega \in \Omega^{2}$ which we can demand to be closed. Alternatively, one may attempt to develop a theory of 'quantum-Lie algebras' and use the 'quantum-Lie bracket' on $L$, thereby avoiding the invertibility assumption. This will not be attempted here, however; for our present purposes we note that in 2 dimensions with the classical differential calculus the Jacobi identity is redundant (similarly, every 2 -form is closed). For our particular exterior algebras the dimensions are the classical ones (so that every 2-form is closed) and one may similarly consider any antisymmetric bivector field as a Poisson structure. Keeping the general form of the above classical Poisson bracket in mind, we now give the explicit form of the quantum Poisson bracket for a natural class of bivector fields in our example.

Proposition 4.7 For the Planck scale Hopf algebra with the standard quantum differential calculus as above, we consider $\Pi$ of the form

$$
\Pi=\left(\eta^{*} \otimes \xi^{*}-\Psi_{L, L}\left(\eta^{*} \otimes \xi^{*}\right)\right) \otimes \pi(g)
$$

for an arbitrary function $\pi(g)$. Then the corresponding quantum Poisson bracket is

$$
\{a, b\}=\pi(g)\left(a_{\xi} b_{\eta}-a_{\eta} b_{\xi}+\imath A\left(a_{\eta} b_{\eta}+\left(a_{\xi}\right)_{\eta} b_{\eta}-\left(a_{\eta}\right)_{\xi} b_{\eta}\right)+(\imath A)^{2}\left(a_{\eta}\right)_{\eta} b_{\eta}\right)
$$

where $a_{\xi}=\partial_{\xi}$ a, etc. In particular, $\pi(g)=\frac{1}{\mathrm{G}}\left(g^{-1}-1\right)$ gives a deformation of the classical Poisson structure in Proposition 4.0.

Proof We first of all use $\mathrm{d} a=\xi \partial_{\xi} a+\eta \partial_{\eta} a$ and the relations of the exterior algebra to obtain

$$
\mathrm{d} a \wedge \mathrm{d} b=\xi \wedge \eta f, \quad f=a_{\xi} b_{\eta}-a_{\eta} b_{\xi}+\imath A\left(a_{\eta} b_{\eta}+\left(a_{\xi}\right)_{\eta} b_{\eta}-\left(a_{\eta}\right)_{\xi} b_{\eta}\right)+(\imath A)^{2}\left(a_{\eta}\right)_{\eta} b_{\eta} .
$$

Now the pairing can be computed as

$$
\begin{aligned}
\{a, b\} & =\langle\Pi, \mathrm{d} a \wedge \mathrm{d} b\rangle=\left\langle\left(\eta^{*} \otimes \xi^{*}-\Psi\left(\eta^{*} \otimes \xi^{*}\right)\right) \pi(g), \xi \wedge \eta f\right\rangle \\
& =\left\langle\eta^{*} \otimes \xi^{*}-\Psi\left(\eta^{*} \otimes \xi^{*}\right), \xi \otimes \pi(g)_{(1)} \triangleright \eta\right\rangle \pi(g)_{(2)} f \\
& =\left\langle\eta^{*} \otimes \xi^{*}, \xi \otimes \pi(g)_{(1)} \triangleright \eta-\Psi, V\left(\xi \otimes \pi(g)_{(1)} \triangleright \eta\right)\right\rangle \pi(g)_{(2)} f \\
& =\left\langle\eta^{*} \otimes \xi^{*}, \xi \otimes \pi(g)_{(1)} \triangleright \eta-\pi(g)_{(1)} \triangleright \eta \otimes \xi\right\rangle \pi(g)_{(2)} f=\left\langle\eta^{*} \otimes \xi^{*}, \xi \otimes \eta\right\rangle \pi(g) f,
\end{aligned}
$$

where we used functoriality of the braiding under the evaluation morphism to deduce

$$
\left.\left\langle\Psi_{L, L}\left(\eta^{*} \otimes \xi^{*}\right), v \otimes w\right)\right\rangle=\left\langle\eta^{*} \otimes \xi^{*}, \Psi_{V, V}(v \otimes w)\right\rangle
$$

for any $v, w \in V$, and then $\Psi_{V, V}(\xi \otimes w)=w_{(1)} \triangleright \xi \otimes w_{(\infty)}=w \otimes \xi$ since $\xi$ is an invariant element of the crossed module. In the last line we used $g \triangleright \eta=\eta+\imath A \xi$ to see that, although $\eta$ is not invariant, the evaluation $\left\langle Y, g^{n} \triangleright \eta\right\rangle=\langle Y, \eta\rangle$ behaves as if it is. In terms of functions $a(g, p)$, $b(g, p)$ we obtain

$$
\begin{aligned}
& \{: a(g, p):,: b(g, p):\} \\
& =\pi(g):\left(g(g-2) a(g, p)+g\left(\frac{\partial}{\partial g}-2 g+3\right) a(g, p+\imath A)+g(g-1) a(g, p+2 \imath A)\right): \\
& \quad \bullet: g \frac{b(g, p+\imath A)-b(g, p)}{\imath A}:
\end{aligned}
$$




$$
-\pi(g): g \frac{a(g, p+\imath A)-a(g, p)}{\imath A}: \bullet:\left(g \frac{\partial}{\partial g} b(g, p+\imath A)+g(b(g, p+\imath A)-b(g, p))\right): .
$$

The classical limit $A \rightarrow 0$ is

$$
\{a(g, p), b(g, p)\}=\pi(g) g^{2}\left(\frac{\partial a}{\partial g} \frac{\partial b}{\partial p}-\frac{\partial a}{\partial p} \frac{\partial b}{\partial g}\right) .
$$

Thus, to get the correct Poisson structure, we need $\pi(g)=\frac{1}{\mathrm{G}}\left(g^{-1}-1\right)$ (note that $\left.-\mathrm{G} \frac{\partial}{\partial x}=g \frac{\partial}{\partial g}\right)$.

Also, if : $h: \in \mathbb{C}[x] \bowtie_{\hbar, G} \mathbb{C}[p]$ is a choice of Hamiltonian then

$$
\begin{aligned}
& \dot{x}=\{x,: h:\}=\frac{\mathrm{G}}{\imath \hbar}:\left(e^{-\frac{x}{\mathrm{G}}}-1\right)\left(h\left(x, p+\frac{\imath \hbar}{\mathrm{G}}\right)-h(x, p)\right): \\
& \left.\dot{p}=\{p,: h:\}=:-\left(e^{-\frac{x}{\mathrm{G}}}-1\right) \frac{\partial}{\partial x} h(x, p+\imath A)\right):
\end{aligned}
$$

are the corresponding quantum Hamilton equations of motion. For a simple concrete example, choosing the Hamiltonian $h(x, p)=\frac{p^{2}}{2 m}+V(x)$ for a free particle of mass $m$ in a potential $V(x)$, we obtain

$$
\dot{x}=\frac{1}{2 m}\left(e^{-\frac{x}{\mathrm{G}}}-1\right)\left(2 p-\frac{\imath \hbar}{\mathrm{G}}\right), \quad \dot{p}=\left(e^{-\frac{x}{\mathrm{G}}}-1\right) \frac{\partial}{\partial x} V(x) .
$$

Standard quantum mechanics (i.e. using the commutator with $h$ ) leads by contrast to

$$
\dot{x}=\frac{\imath}{\hbar}[x, h]=\frac{1}{2 m}\left(e^{-\frac{x}{\mathrm{G}}}-1\right)\left(2 p-\frac{\imath \hbar}{\mathrm{G}} e^{-\frac{x}{\mathrm{G}}}\right), \quad \dot{p}=\frac{\imath}{\hbar}[p, h]=\left(e^{-\frac{x}{\mathrm{G}}}-1\right) \frac{\partial}{\partial x} V(x) .
$$

Thus the quantum Hamiltonian equations of motion reduce to the classical ones when $\hbar \rightarrow 0$ as they should, but also approximate to the conventional quantum mechanical equations of motion in the Planckian strongly gravitational region where $x<<\mathrm{G}$. (We recall that the quantum mechanical evolution in this model approximates flat space when $x>>$ G.) On the other hand, the quantum Hamiltonian equations retain a full (quantum) geometrical interpretation which is lost in conventional quantum mechanics. This suggests a geometrical modification of conventional quantum mechanics.

\section{$5 \quad$ Fourier theory on the Planck scale Hopf algebra}

In this concluding section we make some remarks about the noncommutative Fourier theory which is known to exist on any Hopf algebra equipped with a suitable translation-invariant integral and a suitable exponential element. We recall first the general formulation, which works basically when the Hopf algebra $H$ is finite-dimensional, and in conventions suitable for our particular example. Thus, we require $\int: H \rightarrow k$ such that $\left(\int h_{(1)}\right) h_{(2)}=\left(\int h\right) 1$ for all $h \in H$ (a right-integral) and $\int^{*}: H^{*} \rightarrow k$ such that $\phi_{(1)} \int^{*} \phi_{(2)}=1 \int \phi$ for all $\phi \in H^{*}$ (a left-integral), and we let $\exp =\sum e_{a} \otimes f^{a} \in H \otimes H^{*}$ denote the canonical coevaluation element (here $\left\{e_{a}\right\}$ is a basis of $H$ and $\left\{f^{a}\right\}$ a dual basis). Then the Fourier transform in these conventions is

$$
\mathcal{T}(h)=\left(\int e_{a} h\right) f^{a}, \quad \mathcal{T}^{*}(\phi)=e_{a} \int^{*} f^{a} \phi
$$


and obeys

$$
\mathcal{T} \mathcal{T}^{*}(\phi)=S^{-1} \phi \int e_{a} \int^{*} f^{a}, \quad \mathcal{T}\left(h_{(1)}\left\langle\phi, h_{(2)}\right\rangle\right)=\mathcal{T}(h) S^{-1} \phi, \quad \mathcal{T}^{*}\left(\left\langle\phi_{(1)}, h\right\rangle \phi_{(2)}\right)=S h \mathcal{T}^{*}(\phi) .
$$

These elementary facts are easily proven once one notes that $\left(\int g_{(1)} h\right) g_{(2)}=\left(\int g h_{(1)}\right) S h_{(2)}$ for all $h, g \in H$ and a similar identity on $H^{*}$. See also [5], 21] for more discussion (and the extension to braided groups).

In our case the Planck scale Hopf algebra $\mathbb{C}[x] \bowtie_{\hbar, \mathrm{G}} \mathbb{C}[p]$ is not finite-dimensional and there is, moreover, no purely algebraic integral. For a full treatment one needs to introduce a Hopfvon Neumann algebra setting along the lines in [18] and work with the integral as a weight, or one has to work with a $C^{*}$ algebra setting extended to include unbounded operators. Both of these are nontrivial and beyond our scope here. However, the bicrossproduct form of the Hopf algebra allows one to identify elements as normal ordered versions of ordinary functions $f(x, p)$ and thereby to reduce integration to ordinary integration of ordinary functions, for any class of functions and any topological setting to which the normal ordering extends. Therefore in this section we will initially work formally with $x, p$ as generators (unlike the algebraic setting in the preceeding sections) and proceed to consider formal power series in them; however, what we arrive at in this way is a well-defined deformed Fourier theory on functions on $\mathbb{R}^{2}$ of suitably rapid decay, motivated by the Hopf algebra $\mathbb{C}[x] \triangleleft_{\hbar, \mathrm{G}} \mathbb{C}[p]$ and consistent with any operator algebra setting to which normal ordering extends. This is what we shall outline in this section.

First of all, the bicrossproduct form of the Hopf algebra implies that

$$
\int: f(x, p):=\int_{-\infty}^{\infty} \int_{-\infty}^{\infty} \mathrm{d} x \mathrm{~d} p f(x, p)
$$

is a left-integral on $\mathbb{C}[x] \bowtie_{\hbar, \mathrm{G}} \mathbb{C}[p]$. This is also evident from the explicit form of the rightinvariant derivatives (12)-(13), from which we see that the integrals of $\partial_{x}: f:$ and $\partial_{\eta}: f$ : vanish for suitably decaying $f$. On the other hand the right-integral desired in our preferred conventions for the Fourier theory can be similarly obtained using the left-invariant partial differentials (15)-(16) stated at the end of Section 4.1 one finds

$$
\int: f(x, p):=\int_{-\infty}^{\infty} \int_{-\infty}^{\infty} \mathrm{d} x \mathrm{~d} p e^{\frac{x}{\mathrm{G}}} f(x, p),
$$

which is the right-integral that we shall use. (Although apparently more complicated, the resulting Fourier theory turns out to be more computable in these conventions.)

Next, we recall from [7] [5] that the Planck scale Hopf algebra is essentially self-dual. More precisely, if we let $\bar{x}, \bar{p}$ be dual to the $p, x$ generators in the sense $\left\langle\bar{x}, x^{n} p^{m}\right\rangle=\imath \delta_{n, 0} \delta_{m, 1}$ and $\left\langle\bar{p}, x^{n} p^{m}\right\rangle=\imath \delta_{n, 1} \delta_{m, 0}$, we have an algebraic model of the dual of $\mathbb{C}[x] \triangleleft_{\hbar, \mathrm{G}} \mathbb{C}[p]$ as

$$
\mathbb{C}[\bar{p}] \bowtie_{\frac{1}{\hbar}, \frac{\mathrm{G}}{\hbar}} \mathbb{C}[\bar{x}] \subseteq\left(\mathbb{C}[x] \bowtie_{\hbar, \mathrm{G}} \mathbb{C}[p]\right)^{*},
$$

where

$$
[\bar{p}, \bar{x}]=\frac{\imath}{\hbar}\left(1-e^{-\bar{x} \frac{\hbar}{G}}\right), \quad \Delta \bar{x}=\bar{x} \otimes 1+1 \otimes \bar{x}, \quad \Delta \bar{p}=\bar{p} \otimes 1+e^{-\bar{x} \frac{\hbar}{G}} \otimes \bar{p} .
$$

This is has the same form as $\mathbb{C}[x] \bowtie_{\hbar, \mathrm{G}} \mathbb{C}[p]$ but with different parameter values and with the opposite product and opposite coproduct. On this Hopf algebra we define normal ordering as putting all the $\bar{x}$ to the right and the corresponding left-integral is

$$
\int^{*}: f(\bar{p}, \bar{x}):=\int_{-\infty}^{\infty} \int_{-\infty}^{\infty} \mathrm{d} \bar{p} \mathrm{~d} \bar{x} e^{\bar{x} \frac{\hbar}{\mathrm{G}}} f(\bar{p}, \bar{x}) .
$$


Also from the bicrossproduct form, the canonical element is $[5$

$$
\exp =\sum_{n, m} \frac{1}{n ! m ! \iota^{n+m}} x^{n} p^{m} \otimes \bar{p}^{n} \bar{x}^{m} .
$$

Finally, we will need explicitly the actions [5]

$$
p \triangleright f(x)=\imath \hbar\left(e^{-\frac{x}{G}}-1\right) \frac{\partial}{\partial x} f, \quad f(\bar{x}) \triangleleft \bar{p}=\frac{\imath}{\hbar}\left(e^{-\frac{\hbar}{G} \bar{x}}-1\right) \frac{\partial}{\partial \bar{x}} f
$$

in the bicrossproduct construction and its dual.

Proposition 5.1 The quantum Fourier transform on the Planck scale Hopf algebra is

$$
\mathcal{T}(: f(x, p):)=\int_{-\infty}^{\infty} \int_{-\infty}^{\infty} \mathrm{d} x \mathrm{~d} p e^{-\imath\left(\bar{p}+\frac{\imath}{\mathrm{G}}\right) \cdot x} e^{-\imath \bar{x} \cdot(p+p \triangleright)} f(x, p)
$$

and its dual is

$$
\mathcal{T}^{*}(: f(\bar{p}, \bar{x}):)=\int_{-\infty}^{\infty} \int_{-\infty}^{\infty} \mathrm{d} \bar{p} \mathrm{~d} \bar{x} e^{-\imath \bar{p} \cdot x} e^{-\imath \bar{x} \cdot p} f(\triangleleft \bar{p}+\bar{p}, \bar{x}) e^{\frac{\hbar}{\mathrm{G}} \bar{x}}
$$

where $p \triangleright$ acts only on the functions in $x$ to the right in the integral $(\triangleleft \bar{p}$ acts only on functions in $\bar{x}$ to the left).

Proof We use the reordering equality

$$
: f(p):: h(x):=: e^{p \triangleright \cdot \frac{\partial}{\partial p}} h(x) f(p):=: f(p+p \triangleright) h(x):
$$

in $\mathbb{C}[x] \bowtie_{\hbar, \mathrm{G}} \mathbb{C}[p]$ for functions $f, h$ ( $p \triangleright$ only acts on functions of $x$ ). This follows from the relation $[p, f(x)]=p \triangleright f(x)$ for functions $f(x)$, which is the semidirect product form of the algebra in the bicrossproduct. Hence,

$$
\begin{aligned}
\mathcal{T}(: f(x, p):) & =\sum_{n, m} \frac{1}{n ! m ! \imath^{n+m}} \bar{p}^{n} \bar{x}^{m} \int x^{n} p^{m}: f(x, p): \\
& =\sum_{n, m} \frac{1}{n ! m ! \imath^{n+m}} \bar{p}^{n} \bar{x}^{m} \int: x^{n}(p+p \triangleright)^{m} f(x, p): \\
& =\sum_{n, m} \frac{1}{n ! m ! \imath^{n+m}} \bar{p}^{n} \bar{x}^{m} \int \mathrm{d} x \mathrm{~d} p e^{\frac{x}{G}} x^{n}(p+p \triangleright)^{m} f(x, p) \\
& =\int \mathrm{d} x \mathrm{~d} p e^{-\imath\left(\bar{p}+\frac{\imath}{\mathrm{G}}\right) \cdot x} e^{-\imath \bar{x} \cdot(p+p \triangleright)} f(x, p),
\end{aligned}
$$

where $p \triangleright$ only acts in the powers of $x$ to its right. In $\mathbb{C}[\bar{p}] \bigwedge_{\frac{1}{\hbar}, \frac{G}{\hbar}} \mathbb{C}[\bar{x}]$ we have similarly

$$
: f(\bar{x}):: h(\bar{p}):=: f(\bar{x}) e^{\triangleleft \bar{p} \cdot \frac{\partial}{\partial \bar{p}}} h(\bar{p}):=: f(\bar{x}) h(\triangleleft \bar{p}+p): \text {. }
$$

Hence,

$$
\mathcal{T}^{*}(: f(\bar{p}, \bar{x}):)=\sum_{n, m} \frac{1}{n ! m ! \iota^{n+m}} x^{n} p^{m} \int^{*} \bar{p}^{n} \bar{x}^{m}: f(\bar{p}, \bar{x}):
$$




$$
\begin{aligned}
& =\sum_{n, m} \frac{1}{n ! m ! \imath^{n+m}} x^{n} p^{m} \int^{*}: \bar{p}^{n} \bar{x}^{m} f(\triangleleft \bar{p}+\bar{p}, \bar{x}): \\
& =\sum_{n, m} \frac{1}{n ! m ! \iota^{n+m}} x^{n} p^{m} \int \mathrm{d} \bar{p} \mathrm{~d} \bar{x} \bar{p}^{n} \bar{x}^{m} f(\triangleleft \bar{p}+\bar{p}, \bar{x}) e^{\frac{\hbar}{\mathrm{G}} \bar{x}} \\
& =\int \mathrm{d} \bar{p} \mathrm{~d} \bar{x} e^{-\imath \bar{p} \cdot x} e^{-\imath \bar{x} \cdot p} f(\triangleleft \bar{p}+\bar{p}, \bar{x}) e^{\frac{\hbar}{\mathrm{G}} \bar{x}} .
\end{aligned}
$$

From the properties of the Fourier transform, we see in particular that it turns the (leftinvariant) derivatives $\bar{\partial}_{x}$ and $\bar{\partial}_{\eta}$ in (15)-(16) into multiplication by the corresponding element of the dual. Also, these derivatives become right-handed derivatives $\partial_{\bar{x}}$ and $\partial_{\bar{\eta}}$ on $\mathbb{C}[\bar{p}] \aleph_{\frac{1}{\hbar}, \frac{G}{\hbar}} \mathbb{C}[\bar{x}]$ by identifying it with the opposite algebra and coalgebra to $\mathbb{C}[x] \triangleright_{\frac{1}{\hbar}}, \frac{G}{\hbar} \mathbb{C}[p]$ and making the corresponding notational and parameter changes.

\section{Proposition 5.2}

$$
\begin{gathered}
\mathcal{T}\left(\bar{\partial}_{x} a\right)=\mathcal{T}(a) \imath \bar{p} e^{\frac{\hbar}{\mathrm{G}} \bar{x}} \quad \mathcal{T}\left(\bar{\partial}_{\eta} a\right)=\mathcal{T}(a) \frac{\imath \mathrm{G}}{\hbar}\left(e^{\frac{\hbar}{\mathrm{G}} \bar{x}}-1\right) \\
\mathcal{T}^{*}\left(\partial_{\bar{x}} \phi\right)=\imath p e^{\frac{x}{\mathrm{G}}} \mathcal{T}^{*}(\phi) \quad \mathcal{T}^{*}\left(\partial_{\bar{\eta}} \phi\right)=\imath \mathrm{G}\left(e^{\frac{x}{\mathrm{G}}}-1\right) \mathcal{T}^{*}(\phi) \\
\mathcal{T} \mathcal{T}^{*}(\phi)=(2 \pi)^{2} S^{-1} \phi
\end{gathered}
$$

Proof This is a short computation to identify the partial derivatives as $\bar{\partial}_{x}(a)=a_{(1)}\left\langle-\imath \bar{p}, a_{(2)}\right\rangle$ and $\bar{\partial}_{\eta}(a)=a_{(1)}\left\langle\frac{\imath \mathrm{G}}{\hbar}\left(e^{-\frac{\hbar}{\mathrm{G}} \bar{x}}-1\right), a_{(2)}\right\rangle$, i.e. to identify the corresponding elements of $L$. Similarly, $\partial_{\bar{x}}$ corresponds to $-\imath p$ and $\partial_{\bar{\eta}}$ corresponds to $\imath \mathrm{G}\left(e^{-\frac{x}{G}}-1\right)$ via the right coregular action. One can then verify the analogue of (24) directly in our setting for functions of suitably rapid decay.

Note that when we take the limit $\hbar \rightarrow 0$ the Hopf algebra $\mathbb{C}[\bar{p}] \bowtie_{\frac{1}{\hbar}}, \frac{G}{\hbar} \mathbb{C}[\bar{x}]$ becomes $U\left(b_{+}\right)$or $\kappa$-Minkowski space [8] with the relations

$$
[\bar{p}, \bar{x}]=\frac{\imath}{\mathrm{G}} \bar{x}
$$

(i.e. $\kappa=\frac{\mathrm{G}}{\imath}$ ) regarded as a noncommutative space. Thus,

Corollary 5.3 In the classical limit $\hbar \rightarrow 0$ the Fourier transform becomes

$$
\begin{array}{cc}
\mathcal{T}: C\left(B_{+}\right) \rightarrow U\left(\mathfrak{b}_{+}\right), & \mathcal{T}(: f(x, p):)=\int_{-\infty}^{\infty} \int_{-\infty}^{\infty} \mathrm{d} x \mathrm{~d} p e^{-\imath\left(\bar{p}+\frac{1}{\kappa}\right) \cdot x} e^{-l \bar{x} \cdot p} f(x, p), \\
\mathcal{T}^{*}: U\left(\mathfrak{b}_{+}\right) \rightarrow C\left(B_{+}\right), & \mathcal{T}^{*}(: f(\bar{p}, \bar{x}):)=\int_{-\infty}^{\infty} \int_{-\infty}^{\infty} \mathrm{d} \bar{p} \mathrm{~d} \bar{x} e^{-\imath \bar{p} \cdot x} e^{-\imath \bar{x} \cdot p} f(\triangleleft \bar{p}+\bar{p}, \bar{x})
\end{array}
$$

with $f(\bar{x}) \triangleleft \bar{p}=-\frac{\bar{x}}{\kappa} \frac{\partial}{\partial \bar{x}} f$. Moreover,

$$
\begin{gathered}
\mathcal{T}\left(\bar{\partial}_{x} a\right)=\mathcal{T}(a) \imath \bar{p}, \quad \mathcal{T}\left(\bar{\partial}_{\eta} a\right)=\mathcal{T}(a) \imath \bar{x} \\
\bar{\partial}_{x}(: f(x, p):)=: \frac{\partial}{\partial x} f(x, p)+\frac{\imath p}{\kappa} \frac{\partial}{\partial p} f(x, p):, \quad \bar{\partial}_{\eta}(: f(x, p):)=: \frac{\partial}{\partial p} f(x, p): .
\end{gathered}
$$


The intertwiner properties of $\mathcal{T}^{*}$ in this limit are read from Proposition 5.2 while required right-derivatives simplify to

$$
\partial_{\bar{x}}(: f(\bar{p}, \bar{x}):)=\frac{\partial}{\partial \bar{x}} f(\bar{p}, \bar{x}):, \quad \partial_{\bar{\eta}}(: f(\bar{p}, \bar{x}):)=:-\kappa\left(f\left(\bar{p}-\frac{1}{\kappa}, \bar{x}\right)-f(\bar{p}, \bar{x})\right): .
$$

We also have a dual limit $\hbar, \mathrm{G} \rightarrow \infty$ with $\frac{\mathrm{G}}{\imath \hbar}=\kappa$ constant, where $\mathbb{C}[x] \bowtie_{\hbar, \mathrm{G}} \mathbb{C}[p]$ becomes $U\left(\mathfrak{b}_{-}\right)$(the opposite Lie algebra to $\mathfrak{b}_{+}$) and $\mathbb{C}[\bar{p}] \aleph_{\frac{1}{\hbar}, \frac{G}{\hbar}} \mathbb{C}[\bar{x}]$ becomes $C\left(B_{-}\right)$. We regard the former as another version of $\kappa$-Minkowski space (with opposite commutation relations).

Corollary 5.4 In the limit $\hbar, \mathrm{G} \rightarrow \infty$ with $\frac{\mathrm{G}}{\imath \hbar}=\kappa$ the Fourier transform becomes

$$
\begin{array}{cc}
\mathcal{T}: U\left(\mathfrak{b}_{-}\right) \rightarrow C\left(B_{-}\right), & \mathcal{T}(: f(x, p):)=\int_{-\infty}^{\infty} \int_{-\infty}^{\infty} \mathrm{d} x \mathrm{~d} p e^{-\imath \bar{p} \cdot x} e^{-\imath \bar{x} \cdot(p+p \triangleright)} f(x, p), \\
\mathcal{T}^{*}: C\left(B_{-}\right) \rightarrow U\left(\mathfrak{b}_{-}\right), & \mathcal{T}^{*}(: f(\bar{p}, \bar{x}):)=\int_{-\infty}^{\infty} \int_{-\infty}^{\infty} \mathrm{d} \bar{p} \mathrm{~d} \bar{x} e^{-\imath \bar{p} \cdot\left(x+\frac{1}{\kappa}\right)} e^{-\imath \bar{x} \cdot p} f(\bar{p}, \bar{x})
\end{array}
$$

with $p \triangleright f(x)=-\frac{1}{\kappa} \frac{\partial}{\partial x} f$. Moreover,

$$
\begin{gathered}
\mathcal{T}^{*}\left(\partial_{\bar{x}} \phi\right)=\imath p \mathcal{T}^{*}(\phi), \quad \mathcal{T}^{*}\left(\partial_{\bar{\eta}} \phi\right)=\imath x \mathcal{T}^{*}(\phi), \\
\partial_{\bar{x}}(: f(\bar{x}, \bar{p}):)=: \frac{\partial}{\partial \bar{x}} f(\bar{x}, \bar{p})+\frac{\imath p}{\kappa} \frac{\partial}{\partial \bar{p}} f(\bar{x}, \bar{p}):, \quad \partial_{\bar{\eta}}(: f(\bar{x}, \bar{p}):)=: \frac{\partial}{\partial \bar{p}} f(\bar{x}, \bar{p}):
\end{gathered}
$$

In this limit the intertwiner properties of $\mathcal{T}$ do not simplify (we refer to Proposition 5.2), but the corresponding derivatives become

$$
\bar{\partial}_{x}(: f(x, p):)=: \frac{\partial}{\partial x} f(x, p):, \quad \bar{\partial}_{\eta}(: f(x, p):)=:-\kappa\left(f\left(x, p-\frac{1}{\kappa}\right)-f(x, p)\right): .
$$

Therefore we obtain in fact two versions of Fourier theory on $\kappa$-Minkowski space as two limits of Fourier theory on the Planck scale Hopf algebra. This Hopf algebra, being of self-dual form, has the power to become both a classical but curved phase space (the classical limit) and its dual (the second limit), in addition to the flat space quantum mechanics limit.

There are many further possible developments of the geometry and Fourier theory on the noncommutative phase space in this toy model of Planck scale physics, among them quantum field theory (second quantisation) in a first-order formalism. There is also a physical interpretation of the self-duality as an observable-state duality [0] [20] which should be related to the noncommutative geometric picture above. Finally, we note that there are higher dimensional models of the bicrossproduct form [19] [18] which could be investigated from a similar point of view. These are some directions for further work.

\section{Acknowledgements}

R.O. thanks the German Academic Exchange Service (DAAD) and EPSRC for financial support.

\section{A Direct proofs for crossed modules}

Theorem 2.8 and Proposition 3.5 were deduced somewhat indirectly from our twisting results on bicovariant bimodules and exterior algebras. On the other hand crossed modules ${ }_{H}^{H} \dot{\mathcal{M}}$ have been 
used in a variety of other contexts not related to differential calculi and full direct proofs using conventional Hopf algebra methods may also be useful. For completeness, we provide these here.

Proof of Theorem 2.8 First, we show that $\mathcal{F}_{\chi}$ is a functor, then we verify that it is monoidal. We proceed to check the braiding and finally show that $\mathcal{F}_{\chi}$ is an isomorphism.

(a) $\beta_{\chi}$ is a coaction of $H_{\chi}$ :

$$
\begin{aligned}
(\epsilon \otimes \mathrm{id}) \circ \beta_{\chi}(v) & =\epsilon\left(\chi^{(1)}\left(\chi^{-(1)} \triangleright v\right)_{(1)} \chi^{-(2)}\right) \chi^{(2)} \triangleright\left(\chi^{-(1)} \triangleright v\right)_{(\infty)} \\
& =\epsilon\left(\left(\chi^{-(1)} \triangleright v\right)_{(1)} \chi^{-(2)}\right)\left(\chi^{-(1)} \triangleright v\right)_{(\infty)} \\
& =\epsilon\left(v_{(1)}\right) v_{(\infty)}=v
\end{aligned}
$$

We used the counitality of $\chi$ and $\chi^{-1}$.

$$
\begin{aligned}
& \left(\Delta_{\chi} \otimes \mathrm{id}\right) \circ \beta_{\chi}(v) \\
& =\Delta_{\chi}\left(\chi^{(1)}\left(\chi^{-(1)} \triangleright v\right)_{(1)} \chi^{-(2)}\right) \otimes \chi^{(2)} \triangleright\left(\chi^{-(1)} \triangleright v\right)_{(\infty)} \\
& =\chi^{\prime(1)} \chi_{(1)}^{(1)}\left(\chi^{-(1)} \triangleright v\right)_{(1)(1)} \chi^{-(2)}{ }_{(1)} \chi^{\prime-(1)} \\
& \otimes \chi^{\prime(2)} \chi_{(2)}^{(1)}\left(\chi^{-(1)} \triangleright v\right)_{(1)(2)} \chi^{-(2)}{ }_{(2)} \chi^{\prime-(2)} \otimes \chi^{(2)} \triangleright\left(\chi^{-(1)} \triangleright v\right)_{(\infty)} \\
& =\chi^{\prime(1)} \chi_{(1)}^{(1)}\left(\chi^{-(1)} \triangleright v\right)_{(1)} \chi_{(1)}^{-(2)} \chi^{\prime-(1)} \\
& \otimes \chi^{\prime(2)} \chi_{(2)}^{(1)}\left(\chi^{-(1)} \triangleright v\right)_{(\infty)(1)} \chi^{-(2)}{ }_{(2)} \chi^{\prime-(2)} \otimes \chi^{(2)} \triangleright\left(\chi^{-(1)} \triangleright v\right)_{(\infty)(\infty)} \\
& =\chi^{\prime(1)} \chi_{(1)}^{(1)}\left(\chi_{(1)}^{-(1)} \chi^{\prime-(1)} \triangleright v\right)_{(1)} \chi_{(2)}^{-(1)} \chi^{\prime-(2)} \\
& \otimes \chi^{\prime(2)} \chi_{(2)}^{(1)}\left(\chi_{(1)}^{-(1)} \chi^{\prime-(1)} \triangleright v\right)_{(\infty)(1)} \chi^{-(2)} \otimes \chi^{(2)} \triangleright\left(\chi^{-(1)}{ }_{(1)} \chi^{\prime-(1)} \triangleright v\right)_{(\infty)(\infty)} \\
& =\chi^{\prime(1)} \chi_{(1)}^{(1)} \chi_{(1)}^{-(1)}\left(\chi^{\prime-(1)} \triangleright v\right)_{(1)} \chi^{\prime-(2)} \\
& \otimes \chi^{\prime(2)} \chi_{(2)}^{(1)}\left(\chi_{(2)}^{-(1)} \triangleright\left(\chi^{\prime-(1)} \triangleright v\right)_{(\infty)}\right)_{(1)} \chi^{-(2)} \otimes \chi^{(2)} \triangleright\left(\chi_{(2)}^{-(1)} \triangleright\left(\chi^{\prime-(1)} \triangleright v\right)_{(\infty)}\right)_{(\infty)} \\
& =\chi^{(1)} \chi^{-(1)}\left(\chi^{\prime-(1)} \triangleright v\right)_{(1)} \chi^{\prime-(2)} \\
& \otimes \chi^{\prime(1)} \chi_{(1)}^{(2)}\left(\chi^{-(1)}{ }_{(2)} \triangleright\left(\chi^{\prime-(1)} \triangleright v\right)_{(\infty)}\right)_{(1)} \chi^{-(2)} \otimes \chi^{\prime(2)} \chi_{(2)}^{(2)} \triangleright\left(\chi^{-(1)}{ }_{(2)} \triangleright\left(\chi^{\prime-(1)} \triangleright v\right)_{(\infty)}\right)_{(\infty)} \\
& =\chi^{(1)} \chi^{-(1)}\left(\chi^{\prime-(1)} \triangleright v\right)_{(1)} \chi^{\prime-(2)} \\
& \otimes \chi^{\prime(1)}\left(\chi^{(2)}{ }_{(1)} \chi^{-(1)}{ }_{(2)} \triangleright\left(\chi^{\prime-(1)} \triangleright v\right)_{(\infty)}\right)_{(1)} \chi^{(2)}{ }_{(2)} \chi^{-(2)} \otimes \chi^{\prime(2)} \triangleright\left(\chi_{(2)}^{(2)} \chi^{-(1)}{ }_{(2)} \triangleright\left(\chi^{\prime-(1)} \triangleright v\right)_{(\infty)}\right)_{(\infty)} \\
& =\chi^{(1)}\left(\chi^{\prime-(1)} \triangleright v\right)_{(1)} \chi^{\prime-(2)} \\
& \otimes \chi^{\prime(1)}\left(\chi^{-(1)} \chi^{(2)} \triangleright\left(\chi^{\prime-(1)} \triangleright v\right)_{(\infty)}\right)_{(1)} \chi^{-(2)} \otimes \chi^{\prime(2)} \triangleright\left(\chi^{-(1)} \chi^{(2)} \triangleright\left(\chi^{\prime-(1)} \triangleright v\right)_{(\infty)}\right)_{(\infty)} \\
& =\chi^{(1)}\left(\chi^{\prime-(1)} \triangleright v\right)_{(1)} \chi^{\prime-(2)} \otimes \chi^{\prime(1)}\left(\chi^{-(1)} \triangleright \chi^{(2)} \triangleright\left(\chi^{\prime-(1)} \triangleright v\right)_{(\infty)}\right)_{(1)} \chi^{-(2)} \\
& \otimes \chi^{\prime(2)} \triangleright\left(\chi^{-(1)} \triangleright \chi^{(2)} \triangleright\left(\chi^{\prime-(1)} \triangleright v\right)_{(\infty)}\right)_{(\infty)} \\
& =\chi^{(1)}\left(\chi^{\prime-(1)} \triangleright v\right)_{(1)} \chi^{\prime-(2)} \otimes \beta_{\chi}\left(\chi^{(2)} \triangleright\left(\chi^{\prime-(1)} \triangleright v\right)_{(\infty)}\right) \\
& =\left(\mathrm{id} \otimes \beta_{\chi}\right) \circ \beta_{\chi}(v)
\end{aligned}
$$

We used the crossed module property (5) and the cocycle identity (1) $\cdot \chi^{\prime}$ denotes a second copy of $\chi$.

(b) $\beta_{\chi}$ together with the action obeys the crossed module property in the twisted category:

$$
\begin{aligned}
& h_{<1>} v_{<1>} \otimes h_{<2>} \triangleright v_{<\infty>} \\
& =h_{<1>} \chi^{(1)}\left(\chi^{-(1)} \triangleright v\right)_{(1)} \chi^{-(2)} \otimes h_{<2>} \chi^{(2)} \triangleright\left(\chi^{-(1)} \triangleright v\right)_{(\infty)} \\
& =\chi^{\prime(1)} h_{(1)} \chi^{\prime-(1)} \chi^{(1)}\left(\chi^{-(1)} \triangleright v\right)_{(1)} \chi^{-(2)} \otimes \chi^{\prime(2)} h_{(2)} \chi^{\prime-(2)} \chi^{(2)} \triangleright\left(\chi^{-(1)} \triangleright v\right)_{(\infty)} \\
& =\chi^{\prime(1)} h_{(1)}\left(\chi^{-(1)} \triangleright v\right)_{(1)} \chi^{-(2)} \otimes \chi^{\prime(2)} h_{(2)} \triangleright\left(\chi^{-(1)} \triangleright v\right)_{(\infty)} \\
& =\chi^{(1)}\left(h_{(1)} \triangleright\left(\chi^{-(1)} \triangleright v\right)\right)_{(1)} h_{(2)} \chi^{-(2)} \otimes \chi^{\prime(2)} \triangleright\left(h_{(1)} \triangleright\left(\chi^{-(1)} \triangleright v\right)\right)_{(\infty)} \\
& =\chi^{\prime(1)}\left(h_{(1)} \chi^{-(1)} \triangleright v\right)_{(1)} h_{(2)} \chi^{-(2)} \otimes \chi^{(2)} \triangleright\left(h_{(1)} \chi^{-(1)} \triangleright v\right)_{(\infty)}
\end{aligned}
$$




$$
\begin{aligned}
& =\chi^{\prime(1)}\left(\chi^{\prime-(1)} \chi^{(1)} h_{(1)} \chi^{-(1)} \triangleright v\right)_{(1)} \chi^{\prime-(2)} \chi^{(2)} h_{(2)} \chi^{-(2)} \otimes \chi^{\prime(2)} \triangleright\left(\chi^{\prime-(1)} \chi^{(1)} h_{(1)} \chi^{-(1)} \triangleright v\right)_{(\infty)} \\
& =\chi^{\prime(1)}\left(\chi^{\prime-(1)} \triangleright\left(h_{<1>} \triangleright v\right)\right)_{(1)} \chi^{\prime-(2)} h_{<2>} \otimes \chi^{\prime(2)} \triangleright\left(\chi^{\prime-(1)} \triangleright\left(h_{<1>} \triangleright v\right)\right)_{(\infty)} \\
& =\left(h_{<1>} \triangleright v\right)_{<1>} h_{<2>} \otimes\left(h_{<1>} \triangleright v\right)_{<\infty>}
\end{aligned}
$$

We used the crossed module property in the untwisted category and subscripts $<1>$ etc., for the twisted coproduct and twisted coaction.

(c) To conclude that $\mathcal{F}_{\chi}$ is a functor, we have to show that it maps morphisms to morphisms. Morphisms are module-comodule maps. It is clear that $\mathcal{F}_{\chi}$ maps module maps to module maps since it does not alter the action. It is also easy to see that it maps module-comodule maps to comodule maps. Say $f: V \rightarrow W$ is a morphism in ${ }_{H}^{H} \dot{\mathcal{M}}$. Then

$$
\begin{aligned}
\beta_{\chi}(f(v)) & =\chi^{(1)}\left(\chi^{-(1)} \triangleright f(v)\right)_{(1)} \chi^{-(2)} \otimes \chi^{(2)} \triangleright\left(\chi^{-(1)} \triangleright f(v)\right)_{(\infty)} \\
& =\chi^{(1)}\left(f\left(\chi^{-(1)} \triangleright v\right)\right)_{(1)} \chi^{-(2)} \otimes \chi^{(2)} \triangleright\left(f\left(\chi^{-(1)} \triangleright v\right)\right)_{(\infty)} \\
& =\chi^{(1)}\left(\chi^{-(1)} \triangleright v\right)_{(1)} \chi^{-(2)} \otimes \chi^{(2)} \triangleright f\left(\left(\chi^{-(1)} \triangleright v\right)_{(\infty)}\right) \\
& =\chi^{(1)}\left(\chi^{-(1)} \triangleright v\right)_{(1)} \chi^{-(2)} \otimes f\left(\chi^{(2)} \triangleright\left(\chi^{-(1)} \triangleright v\right)_{(\infty)}\right) \\
& =(\mathrm{id} \otimes f) \circ \beta_{\chi}(v),
\end{aligned}
$$

as required.

(d) We proceed to show that $\mathcal{F}_{\chi}$ is monoidal. The associativity property of $c_{\chi}$ clearly reduces to $\chi$ a cocycle, and invertibility reduces to $\chi$ invertible. Naturality of $c_{\chi}$ is also immediate from its stated form. It remains to verify that $c_{\chi}: \mathcal{F}_{\chi}(V) \odot_{\chi} \mathcal{F}_{\chi}(W) \rightarrow \mathcal{F}_{\chi}(V \odot W)$ is indeed a morphism in ${ }_{H_{\chi}}^{H_{\chi}} \dot{\mathcal{M}}$. For clarity we denote the tensor product in ${ }_{H}^{H_{H}} \dot{\mathcal{M}}$ by $\odot$ and that in ${ }_{H_{\chi}}^{H_{\chi}} \dot{\mathcal{M}}$ by $\odot_{\chi}$. For the action of $H_{\chi}$ (which coincides with that of $H$ ) we have

$$
\begin{aligned}
h \triangleright c_{\chi}\left(v \odot_{\chi} w\right) & =h \triangleright\left(\chi^{-(1)} \triangleright v \odot \chi^{-(2)} \triangleright w\right) \\
& =h_{(1)} \chi^{-(1)} \triangleright v \odot h_{(2)} \chi^{-(2)} \triangleright w \\
& =c_{\chi}\left(\chi^{(1)} h_{(1)} \chi^{-(1)} \triangleright v \odot_{\chi} \chi^{(2)} h_{(2)} \chi^{-(2)} \triangleright w\right) \\
& =c_{\chi}\left(h_{<1>} \triangleright v \odot_{\chi} h_{<2>} \triangleright w\right) \\
& =c_{\chi}\left(h \triangleright\left(v \odot_{\chi} w\right)\right) .
\end{aligned}
$$

For the coaction, we have

$$
\begin{aligned}
& \beta_{\chi} \circ c_{\chi}\left(v \odot_{\chi} w\right) \\
&=\beta_{\chi}\left(\chi^{\prime-(1)} \triangleright v \odot \chi^{\prime-(2)} \triangleright w\right) \\
&= \chi^{(1)}\left(\chi^{-(1)} \triangleright\left(\chi^{\prime-(1)} \triangleright v \odot \chi^{\prime-(2)} \triangleright w\right)\right)_{(1)} \chi^{-(2)} \\
& \otimes \chi^{(2)} \triangleright\left(\chi^{-(1)} \triangleright\left(\chi^{\prime-(1)} \triangleright v \odot \chi^{\prime-(2)} \triangleright w\right)\right)_{(\infty)} \\
&= \chi^{(1)}\left(\chi^{-(1)}{ }_{(1)} \chi^{\prime-(1)} \triangleright v \odot \chi^{-(1)}{ }_{(2)} \chi^{\prime-(2)} \triangleright w\right)_{(1)} \chi^{-(2)} \\
& \otimes \chi^{(2)} \triangleright\left(\chi^{-(1)}{ }_{(1)} \chi^{\prime-(1)} \triangleright v \odot \chi^{-(1)}{ }_{(2)} \chi^{\prime-(2)} \triangleright w\right)_{(\infty)} \\
&= \chi^{(1)}\left(\chi^{-(1)}{ }_{(1)} \chi^{\prime-(1)} \triangleright v\right)_{(1)}\left(\chi^{-(1)}{ }_{(2)} \chi^{\prime-(2)} \triangleright w\right)_{(1)} \chi^{-(2)} \\
& \otimes \chi^{(2)} \triangleright\left(\left(\chi^{-(1)}{ }_{(1)} \chi^{\prime-(1)} \triangleright v\right)_{(\infty)} \odot\left(\chi_{(1)}^{-(1)} \chi^{\prime-(2)} \triangleright w\right)_{(\infty)}\right) \\
&= \chi^{(1)}\left(\chi^{-(1)}{ }_{(1)} \chi^{\prime-(1)} \triangleright v\right)_{(1)}\left(\chi^{-(1)}{ }_{(2)} \chi^{\prime-(2)} \triangleright w\right)_{(1)} \chi^{-(2)} \\
& \otimes \chi^{(2)}{ }_{(1)} \triangleright\left(\chi^{-(1)}{ }_{(1)} \chi^{\prime-(1)} \triangleright v\right)_{(\infty)} \odot \chi^{(2)}{ }_{(2)} \triangleright\left(\chi^{-(1)}{ }_{(2)} \chi^{\prime-(2)} \triangleright w\right)_{(\infty)} \\
&= \chi^{(1)}\left(\chi^{-(1)} \triangleright v\right)_{(1)}\left(\chi^{-(2)}{ }_{(1)} \chi^{\prime-(1)} \triangleright w\right)_{(1)} \chi^{-(2)}{ }_{(2)} \chi^{\prime-(2)} \\
& \otimes \chi^{(2)}{ }_{(1)} \triangleright\left(\chi^{-(1)} \triangleright v\right)_{(\infty)} \odot \chi^{(2)}{ }_{(2)} \triangleright\left(\chi^{-(2)}{ }_{(1)} \chi^{\prime-(1)} \triangleright w\right)_{(\infty)} \\
&= \chi^{(1)}\left(\chi^{-(1)} \triangleright v\right)_{(1)} \chi^{-(2)}{ }_{(1)}\left(\chi^{\prime-(1)} \triangleright w\right)_{(1)} \chi^{\prime-(2)}
\end{aligned}
$$




$$
\begin{aligned}
& \otimes \chi^{(2)}{ }_{(1)} \triangleright\left(\chi^{-(1)} \triangleright v\right)_{(\infty)} \odot \chi^{(2)}{ }_{(2)} \chi^{-(2)}{ }_{(2)} \triangleright\left(\chi^{\prime-(1)} \triangleright w\right)_{(\infty)} \\
= & \chi^{(1)}\left(\chi^{-(1)} \triangleright v\right)_{(1)} \chi^{-(2)}{ }_{(1)} \chi^{\prime \prime-(1)} \chi^{\prime(1)}\left(\chi^{\prime-(1)} \triangleright w\right)_{(1)} \chi^{\prime-(2)} \\
& \otimes \chi^{(2)}{ }_{(1)} \triangleright\left(\chi^{-(1)} \triangleright v\right)_{(\infty)} \odot \chi^{(2)}{ }_{(2)} \chi^{-(2)}{ }_{(2)} \chi^{\prime \prime-(2)} \chi^{\prime(2)} \triangleright\left(\chi^{\prime-(1)} \triangleright w\right)_{(\infty)} \\
= & \chi^{(1)}\left(\chi^{-(1)}{ }_{(1)} \chi^{\prime \prime-(1)} \triangleright v\right)_{(1)} \chi^{-(1)}{ }_{(2)} \chi^{\prime \prime-(2)} \chi^{(1)}\left(\chi^{\prime-(1)} \triangleright w\right)_{(1)} \chi^{\prime-(2)} \\
& \otimes \chi^{(2)}{ }_{(1)} \triangleright\left(\chi^{-(1)}{ }_{(1)} \chi^{\prime \prime-(1)} \triangleright v\right)_{(\infty)} \odot \chi^{(2)}{ }_{(2)} \chi^{-(2)} \chi^{\prime(2)} \triangleright\left(\chi^{\prime-(1)} \triangleright w\right)_{(\infty)} \\
= & \chi^{(1)} \chi^{-(1)}{ }_{(1)}\left(\chi^{\prime \prime-(1)} \triangleright v\right)_{(1)} \chi^{\prime \prime-(2)} \chi^{\prime(1)}\left(\chi^{\prime-(1)} \triangleright w\right)_{(1)} \chi^{\prime-(2)} \\
& \otimes \chi^{(2)}{ }_{(1)} \chi^{-(1)}{ }_{(2)} \triangleright\left(\chi^{\prime \prime-(1)} \triangleright v\right)_{(\infty)} \odot \chi^{(2)}{ }_{(2)} \chi^{-(2)} \chi^{\prime(2)} \triangleright\left(\chi^{\prime-(1)} \triangleright w\right)_{(\infty)} \\
= & \chi^{(1)}\left(\chi^{\prime \prime}{ }_{(1)} \triangleright v\right)_{(1)} \chi^{\prime \prime-(2)} \chi^{\prime(1)}\left(\chi^{\prime-(1)} \triangleright w\right)_{(1)} \chi^{\prime-(2)} \\
& \otimes \chi^{-(1)} \chi^{(2)} \triangleright\left(\chi^{\prime \prime-(1)} \triangleright v\right)_{(\infty)} \odot \chi^{-(2)} \chi^{\prime(2)} \triangleright\left(\chi^{\prime-(1)} \triangleright w\right)_{(\infty)} \\
= & \left(\mathrm{id} \otimes c_{\chi}\right)\left(\chi^{(1)}\left(\chi^{\prime \prime-(1)} \triangleright v\right)_{(1)} \chi^{\prime \prime-(2)} \chi^{\prime(1)}\left(\chi^{\prime-(1)} \triangleright w\right)_{(1)} \chi^{\prime-(2)}\right. \\
& \left.\otimes \chi^{(2)} \triangleright\left(\chi^{\prime \prime-(1)} \triangleright v\right)_{(\infty)} \odot_{\chi} \chi^{\prime(2)} \triangleright\left(\chi^{\prime-(1)} \triangleright w\right)_{(\infty)}\right) \\
= & \left(i d \otimes c_{\chi}\right)\left(v_{<1>} w_{<1>} \otimes v_{<\infty>} \odot_{\chi} w_{<\infty>}\right) \\
= & \left(i d \otimes c_{\chi}\right) \circ \beta_{\chi}\left(v \odot_{\chi} w\right) .
\end{aligned}
$$

We used the crossed module property and the cocycle identities as before, and $\chi^{\prime \prime}$ denotes a third copy of $\chi$.

(e) We next show that $\mathcal{F}_{\chi}$ preserves the braiding. Thus,

$$
\begin{aligned}
c_{\chi} \circ \Psi_{\chi}\left(v \odot_{\chi} w\right) & =c_{\chi}\left(v_{<1>} \triangleright w \odot_{\chi} v_{<\infty>}\right) \\
& =c_{\chi}\left(\chi^{(1)}\left(\chi^{-(1)} \triangleright v\right)_{(1)} \chi^{-(2)} \triangleright w \odot_{\chi} \chi^{(2)} \triangleright\left(\chi^{-(1)} \triangleright v\right)_{(\infty)}\right) \\
& =\left(\chi^{-(1)} \triangleright v\right)_{(1)} \chi^{-(2)} \triangleright w \odot\left(\chi^{-(1)} \triangleright v\right)_{(\infty)} \\
& =\Psi\left(\chi^{-(1)} \triangleright v \odot \chi^{-(2)} \triangleright w\right) \\
& =\Psi \circ c_{\chi}\left(v \odot_{\chi} w\right) .
\end{aligned}
$$

(f) It remains to be shown that $\mathcal{F}_{\chi}$ is an isomorphism. The inverse operation to the twisting by $\chi$ is twisting by $\chi^{-1}$; we verify that the coaction twisted by $\chi$ and then twisted by $\chi^{-1}$ is the original coaction. Thus,

$$
\begin{aligned}
\left(\beta_{\chi}\right)_{\chi^{-1}}(v) & =\chi^{-(1)}\left(\chi^{(1)} \triangleright v\right)_{<1>} \chi^{(2)} \otimes \chi^{-(2)} \triangleright\left(\chi^{(1)} \triangleright v\right)_{<\infty>} \\
& =\chi^{-(1)} \chi^{\prime(1)}\left(\chi^{\prime-(1)} \chi^{(1)} \triangleright v\right)_{(1)} \chi^{\prime-(2)} \chi^{(2)} \otimes \chi^{-(2)} \chi^{(2)} \triangleright\left(\chi^{\prime-(1)} \chi^{(1)} \triangleright v\right)_{(\infty)} \\
& =v_{(1)} \otimes v_{(\infty)}=\beta(v) .
\end{aligned}
$$

For the monoidal structure, one sees immediately that $c_{\chi^{-1}} \circ c_{\chi}$ is the identity transformation.

Theorem 2.7 can likewise be proven directly or else be obtained by dualisation of Theorem 2.8 using conventional methods.

Proof of the intertwiner property of $\alpha$ in Proposition 3.5 in $H_{\chi}$ setting We give the result here in the same coproduct twist setting as Theorem 2.8 proven above (the version in Proposition 3.5 is the dual of this and can be obtained by the same methods or by dualisation of the proof.) Thus, for a Hopf algebra $H$ viewed in ${ }_{H}^{H} \dot{\mathcal{M}}$ by the coproduct (the regular coaction) and adjoint action, and a cocycle $\chi \in H \otimes H$ we show that

$$
\alpha: \mathcal{F}_{\chi}(H) \rightarrow H_{\chi}, \quad \alpha(h)=\left(\chi^{-(1)} \triangleright h\right) \chi^{-(2)}
$$


is an isomorphism of crossed modules, where $H_{\chi}$ is viewed in ${ }_{H_{\chi}}^{H_{\chi}} \dot{\mathcal{M}}$ by its coproduct $\Delta_{\chi}$ and its adjoint action. In fact, that the actions are intertwined is known from [6] (in another context) so we need only to show that the coactions are intertwined.

On the one hand, writing $\beta_{\chi}$ for the coaction induced by Theorem 2.8 on $\mathcal{F}_{\chi}(H)$ by transforming the regular coaction, we have

$$
(\mathrm{id} \otimes \alpha) \beta_{\chi}(h)=\chi^{(1)}\left(\chi^{-(1)} \triangleright h\right)_{(1)} \chi^{-(2)} \otimes\left(\chi^{\prime-(1)} \chi^{(2)} \triangleright\left(\chi^{-(1)} \triangleright h\right)_{(2)}\right) \chi^{\prime-(2)} .
$$

We require this to coincide with

$$
\begin{aligned}
\Delta_{\chi} \alpha(h) & =\chi^{(1)}\left(\chi^{-(1)} \triangleright h\right)_{(1)} \chi^{-(2)}{ }_{(1)} \chi^{-(1)} \otimes \chi^{(2)}\left(\chi^{-(1)} \triangleright h\right)_{(2)} \chi_{(2)}^{-(2)} \chi^{\prime-(2)} \\
& =\chi^{(1)}\left(\chi^{-(1)}{ }_{(1)} \chi^{\prime-(1)} \triangleright h\right)_{(1)} \chi^{-(1)}{ }_{(2)} \chi^{\prime-(2)} \otimes \chi^{(2)}\left(\chi^{-(1)}{ }_{(1)} \chi^{-(1)} \triangleright h\right)_{(2)} \chi^{-(2)} \\
& =\chi^{(1)} \chi^{-(1)}\left({ }_{(1)}\left(\chi^{\prime-(1)} \triangleright h\right)_{(1)} \chi^{\prime-(2)} \otimes \chi^{(2)}\left(\chi^{-(1)}{ }_{(2)} \triangleright\left(\chi^{\prime-(1)} \triangleright h\right)_{(2)}\right) \chi^{-(2)}\right.
\end{aligned}
$$

using the cocycle axiom for $\chi$ and then the crossed module axiom. Comparing these expressions and substituting the quantum group adjoint action of $H$ for $\triangleright$ we see that these expression coincide in view of the identity

$$
\chi^{(1)} \otimes \chi_{(1)}^{-(1)} \chi_{(1)}^{(2)} \otimes\left(S \chi_{(2)}^{(2)}\right)\left(S \chi_{(2)}^{-(1)}\right) \chi^{-(2)}=\chi^{(1)} \chi_{(1)}^{-(1)} \otimes \chi^{(2)} \chi_{(2)}^{-(1)} \otimes\left(S \chi_{(3)}^{-(1)}\right) \chi^{-(2)} .
$$

This is equivalent (by using the cocycle condition (11) on the left hand side repeatedly) to

$$
\chi_{(1)}^{(1)} \otimes \chi_{(2)}^{(1)} \otimes\left(S\left(\chi^{\prime-(1)} \chi^{(2)}\right)\right) \chi^{\prime-(2)}=\chi_{(1)}^{-(1)} \otimes \chi_{(2)}^{-(1)} \otimes\left(S \chi^{-(1)}\right) \chi^{-(2)}
$$

which reduces to

$$
\chi^{(1)} \otimes\left(S\left(\chi^{\prime-(1)} \chi^{(2)}\right)\right) \chi^{\prime-(2)}=\chi^{-(1)} \otimes\left(S \chi_{(3)}^{-(1)}\right) \chi^{-(2)} .
$$

This identity is readily proven from the properties of $U^{-1}=\left(S \chi^{-(1)}\right) \chi^{-(2)}$ in [5] using the cocycle condition. The inverse of the map $\alpha$ is also readily supplied by similar means, so it forms an isomorphism of crossed modules.

\section{References}

[1] S. Majid. Classification of bicovariant differential calculi. J. Geom. Phys., 25:119-140, 1998.

[2] E. Beggs and S. Majid. Quasitriangular and differential structures on bicrossproduct Hopf algebras. Preprint, Damtp/96-97, 1996.

[3] V.G. Drinfeld. Hopf algebras and the quantum Yang-Baxter equations. Sov. Math. Dokl., 32:254-258, 1985.

[4] V.G. Drinfeld. QuasiHopf algebras. Leningrad Math. J., 1:1419-1457, 1990.

[5] S. Majid. Foundations of Quantum Group Theory. Cambridge Univeristy Press, 1995.

[6] D.I. Gurevich and S. Majid. Braided groups of Hopf algebras obtained by twisting. Pac. J. Math., 162:27-44, 1994. 
[7] S. Majid. Hopf algebras for physics at the Planck scale. J. Classical and Quantum Gravity, 5:1587-1606, 1988.

[8] S. Majid and H. Ruegg. Bicrossproduct structure of the $\kappa$-Poincaré group and noncommutative geometry. Phys. Lett. B, 334:348-354, 1994.

[9] F. Bayen, M. Flato, C. Frondsdal, A. Lichnerowicz, and D. Sternheimer. Deformation theory and quantisation i,ii. Ann. Phys., 111:61-151, 1978.

[10] R. Oeckl. Classification of differential calculi on $U_{q}\left(b_{+}\right)$, classical limits, and duality. Preprint, Damtp-1998-86, math.QA/9807097.

[11] V.G. Drinfeld. Quantum groups. In A. Gleason, editor, Proceedings of the ICM, pages 798-820, Rhode Island, 1987. AMS.

[12] D. Radford. The structure of Hopf algebras with a projection. J. Algebra, 92:322-347, 1985.

[13] D.N. Yetter. Quantum groups and representations of monoidal categories. Math. Proc. Camb. Phil. Soc., 108:261-290, 1990.

[14] S.L. Woronowicz. Differential calculus on compact matrix pseudogroups (quantum groups). Commun. Math. Phys., 122:125-170, 1989.

[15] T. Brzeziński. Remarks on bicovariant differential calculi and exterior Hopf algebras. Lett. Math. Phys., 27:287, 1993.

[16] R. Kerner. $Z_{3}$-graded algebras and the cubic root of the supersymmetry transformations. J. Math. Phys., 33:403-411, 1992.

[17] S. Majid. q-Euclidean space and quantum Wick rotation by twisting. J. Math. Phys., 35:5025-5034, 1994.

[18] S. Majid. Hopf-von Neumann algebra bicrossproducts, Kac algebra bicrossproducts, and the classical Yang-Baxter equations. J. Funct. Analysis, 95:291-319, 1991. From PhD Thesis, Harvard, 1988.

[19] S. Majid. Matched pairs of Lie groups associated to solutions of the Yang-Baxter equations. Pac. J. Math., 141:311-332, 1990. From PhD Thesis, Harvard, 1988.

[20] S. Majid. Duality principle and braided geometry. In Strings and Symmetries, volume 447 of Lec. Notes in Phys., pages 125-144. Springer, 1995.

[21] A. Kempf and S. Majid. Algebraic $q$-integration and Fourier theory on quantum and braided spaces. J. Math. Phys., 35:6802-6837, 1994. 